ARTICLE

Received 8 Jul 2015 | Accepted 6 Oct 2015 | Published 10 Nov 2015

DOI: $10.1038 /$ ncomms 9808 OPEN

\title{
Complementation between polymerase- and exonuclease-deficient mitochondrial DNA polymerase mutants in genomically engineered flies
}

\author{
Ana Bratic ${ }^{1, \star}$, Timo E.S. Kauppila, ${ }^{1, \star}$, Bertil Macao ${ }^{2}$, Sebastian Grönke ${ }^{3}$, Triinu Siibak², James B. Stewart ${ }^{1}$, \\ Francesca Baggio', Jacqueline Dols ${ }^{3}$, Linda Partridge ${ }^{3}$, Maria Falkenberg ${ }^{2}$, Anna Wredenberg ${ }^{4}$ \\ \& Nils-Göran Larsson ${ }^{1,4}$
}

Replication errors are the main cause of mitochondrial DNA (mtDNA) mutations and a compelling approach to decrease mutation levels would therefore be to increase the fidelity of the catalytic subunit ( $\mathrm{POL} \gamma \mathrm{A})$ of the mtDNA polymerase. Here we genomically engineer the tamas locus, encoding fly POL $\gamma \mathrm{A}$, and introduce alleles expressing exonuclease- $\left(\mathrm{exo}^{-}\right)$and polymerase-deficient ( $\mathrm{pol}^{-}$) $\mathrm{POL} \gamma \mathrm{A}$ versions. The exo- mutant leads to accumulation of point mutations and linear deletions of mtDNA, whereas pol- mutants cause mtDNA depletion. The mutant tamas alleles are developmentally lethal but can complement each other in trans resulting in viable flies with clonally expanded mtDNA mutations. Reconstitution of human mtDNA replication in vitro confirms that replication is a highly dynamic process where POL $\gamma \mathrm{A}$ goes on and off the template to allow complementation during proofreading and elongation. The created fly models are valuable tools to study germ line transmission of mtDNA and the pathophysiology of POL $\gamma \mathrm{A}$ mutation disease.

\footnotetext{
${ }^{1}$ Department of Mitochondrial Biology, Max Planck Institute for Biology of Ageing, Joseph-Stelzmann-Strasse 9b, Cologne D-50931, Germany. ${ }^{2}$ Department of Medical Biochemistry and Cell Biology, Institute of Biomedicine, University of Gothenburg, Medicinaregatan 9A, Gothenburg SE-40530, Sweden.

${ }^{3}$ Department of Biological Mechanisms of Ageing, Max Planck Institute for Biology of Ageing, Joseph-Stelzmann-Strasse 9b, Cologne D-50931, Germany.

${ }^{4}$ Department of Laboratory Medicine, Karolinska Institutet, Stockholm SE-17177, Sweden. ${ }^{\star}$ These authors contributed equally to this work. Correspondence and requests for materials should be addressed to A.W. (email: anna.wredenberg@ki.se) or to N.-G.L. (email: larsson@age.mpg.de).
} 
$\mathrm{M}$ utations of mitochondrial DNA (mtDNA) are an important cause of human disease and are heavily implicated in the ageing process, consistent with the essential role for mtDNA in maintaining oxidative phosphorylation. MtDNA is fully coated with the mitochondrial transcription factor A (TFAM) protein and compacted into nucleoids ${ }^{1,2}$, which must be at least partly unpackaged to allow mtDNA replication ${ }^{3}$. The metazoan $\mathrm{mtDNA}$ is replicated by DNA polymerase gamma $(\mathrm{POL} \gamma)^{4}$, which belongs to the family A DNA polymerases, as does DNA polymerase I of Escherichia coli ${ }^{5}$. It consists of the catalytic (POL $\gamma \mathrm{A})$ and the accessory subunits $(\mathrm{POL} \gamma \mathrm{B})$, which interact to form a heterodimer in insects ${ }^{6}$ and a heterotrimer in mammals ${ }^{7}$. A minimal mammalian mtDNA replisome, consisting of POL $\gamma$, TWINKLE DNA helicase and mitochondrial single-stranded DNA-binding protein (mtSSB), can synthesize DNA strands longer than $15 \mathrm{~kb}$ in length, thus showing that a single initiation event is sufficient to replicate one strand of $\mathrm{mtDNA}^{8}$. Mouse knockouts (KOs) for POL $\gamma \mathrm{A}^{9}$, POL $\gamma \mathrm{B}^{10}$ and TWINKLE ${ }^{11}$ have shown that all are essential for mtDNA maintenance and embryo survival.

The mode of mammalian mtDNA replication is debated and observations of replication intermediates by electron microscopy or two-dimensional (2D) neutral-neutral agarose gel electrophoresis have been interpreted to support either a strand-asynchronous or a strand-synchronous model ${ }^{12}$. The strand-asynchronous model proposes that leading (heavy $(\mathrm{H})$ ) strand replication initiates at a defined origin of replication $\left(\mathrm{O}_{\mathrm{H}}\right)$ in the control region. Once initiated the leading strand replication continues two-thirds around the circular genome until the origin of lagging (light $(\mathrm{L})$ ) strand replication $\left(\mathrm{O}_{\mathrm{L}}\right)$ is activated and lagging strand replication ensues ${ }^{13}$. Transcription by the mitochondrial RNA polymerase (POLRMT) at the light strand promoter provides the RNA primers necessary for initiation of replication at $\mathrm{O}_{\mathrm{H}}$ and POLRMT is also responsible for providing RNA primers for initiation of replication at $\mathrm{O}_{\mathrm{L}}{ }^{14,15}$. Consistently, KO mice lacking POLRMT develop severe mtDNA depletion causing embryonic lethality ${ }^{16}$. The importance of light strand promoter, $\mathrm{O}_{\mathrm{H}}$ and $\mathrm{O}_{\mathrm{L}}$ is further underscored by the finding that replication-competent mtDNAs, which harbour large deletions in some forms of human mitochondrial disease, always retain these sequences $^{17,18}$. In addition, an in vivo saturation mutagenesis approach in the mouse has shown that $\mathrm{O}_{\mathrm{L}}$ is indispensable for mtDNA maintenance ${ }^{14}$. Further support for the strand asymmetric model was recently presented in a study showing that $\mathrm{mtSSB}$ binds single-stranded mtDNA in a pattern consistent with initiation of replication at $\mathrm{O}_{\mathrm{H}}$ and $\mathrm{O}_{\mathrm{L}}{ }^{19}$.

The occurrence of mtDNA mutations has traditionally been attributed to damage ${ }^{20}$, but several lines of evidence suggest that replication errors may in fact be the main source of mtDNA mutations in mammals ${ }^{21-23}$ and flies ${ }^{24}$. Consistently, mutations in the POL $\gamma A, P O L \gamma B$ and PEO1 (TWINKLE) genes are important causes of human disease ${ }^{25}$. More than 150 different pathogenic mutations have been reported in the POL $\gamma A$ gene and the majority of these causes either a severe depletion of mtDNA or the formation of large deletions. Such deletions can accumulate to high levels because mtDNA is replicated throughout the cell cycle in dividing cells and also undergoes replication in postmitotic cells ${ }^{26}$. Similarly, point mutations of mtDNA can accumulate over time to high levels and affect respiratory chain function and cell viability ${ }^{27}$.

The mtDNA is strictly maternally inherited in mammals and transmitted without any apparent germline recombination ${ }^{28}$. This asexual mode of transmission is predicted to lead to accumulation of deleterious mutations over time ${ }^{29}$. Characterization of mtDNA sequence variation in animal models $^{21,30-32}$, human populations ${ }^{33}$ and wild animals ${ }^{34}$ shows clear evidence of purifying selection. There is likely more than one mechanism that prevents the accumulation of mtDNA mutations between generations ${ }^{29}$. First, the bottleneck mechanism in the maternal mammalian germline can shift the mtDNA genotype in just a few generations and can thereby remove mutations from maternal lineages ${ }^{35-39}$. Second, there is very strong purifying selection against mutations that cause amino acid substitutions in mtDNA-encoded proteins ${ }^{32,40}$. Third, there is a selection against high levels of pathogenic mutations in transfer RNA genes post fertilization ${ }^{30}$. Fourth, females with high levels of mtDNA mutations in the germline have reduced fecundity ${ }^{41}$, which will decrease the risk of transmission of mtDNA mutations.

Given the substantial impact that mtDNA mutations have on human disease and ageing ${ }^{27}$, there is a strong interest in developing animal models that have increased or decreased levels of mtDNA mutations. The mtDNA mutator mice are homozygous for a knock-in mutation that leads to the expression of POL $\gamma \mathrm{A}$ with a severely impaired proofreading during mtDNA replication. These mice develop a premature ageing syndrome and have a shortened life $\operatorname{span}^{42,43}$. Furthermore, mouse strains containing only a subset of all different mtDNA mutations present in mtDNA mutator mice can be derived by breeding and are valuable tools to study mtDNA transmission in the germline $e^{21,30,41}$. While mutator variants with decreased accuracy have been generated for many different DNA polymerases, only a handful of alterations are known to make DNA polymerases more accurate. High-fidelity DNA polymerases have been studied in unicellular organisms, for example, DNA polymerase I in E. coli $^{44}$ and MIP1 (homolog of POL $\gamma \mathrm{A}$ ) in Saccharomyces cerevisiae $^{45}$, but not yet in metazoans. A critical question concerning the role for mtDNA mutations in ageing is to determine whether a decreased mtDNA mutation rate would prolong life span. It is still elusive whether it is possible to engineer enzymes surpassing the accuracy of wild-type (WT) metazoan POL $\gamma \mathrm{A}$ in vivo. To address this issue, we performed a detailed biochemical characterization of exonuclease-deficient (exo ${ }^{-}$, low fidelity) and polymerase-deficient ( $\mathrm{pol}^{-}$, high fidelity) variants of human POL $\gamma \mathrm{A}$ (HsPOL $\gamma \mathrm{A})$ in vitro and created genomically engineered fruit flies expressing the corresponding alleles of fruit fly POL $\gamma$ A (DmPOL $\gamma \mathrm{A})$. Homozygous expression of an exo ${ }^{-}$DmPOL $\gamma \mathrm{A}$ variant in flies leads to accumulation of point mutations and linear deletions of mtDNA, whereas the high-fidelity variants cause mtDNA depletion, which in both situations result in larval lethality. Unexpectedly, exo ${ }^{-}$and $\mathrm{pol}^{-}$ versions of POL $\gamma \mathrm{A}$ complemented each other in trans, resulting in apparently normal compound heterozygous flies with clonally expanded mtDNA mutations. Reconstitution of human mtDNA replication in vitro confirmed that mtDNA replication is a highly dynamic process where HsPOL $\gamma \mathrm{A}$ goes on and off the template to allow complementation during proofreading and elongation.

\section{Results}

HsPOL $\gamma \mathrm{A}$ pol $^{-}$mutants have increased exonuclease activity. Two amino acid substitutions (Q849A and H881A) in E. coli DNA polymerase I are known to cause an antimutator effect during DNA replication ${ }^{44}$. As both of these amino acids are highly conserved in family A polymerases and thus present in both DmPOL $\gamma \mathrm{A}$ (Q1009A and H1038A) and HsPOL $\gamma \mathrm{A}$ (Q1102A and H1134A; Supplementary Fig. 1), we engineered recombinant DmPOL $\gamma \mathrm{A}$ and HsPOL $\gamma \mathrm{A}$ proteins and tested their effects on DNA replication in vitro. In addition, we chose to introduce a mutation affecting the exonuclease domain of POL $\gamma$ A (DmD263A, HsD274A) because such a substitution has previously been shown to exert a mtDNA mutator phenotype in budding yeast ${ }^{46}$ and mice ${ }^{42,43}$. 
a

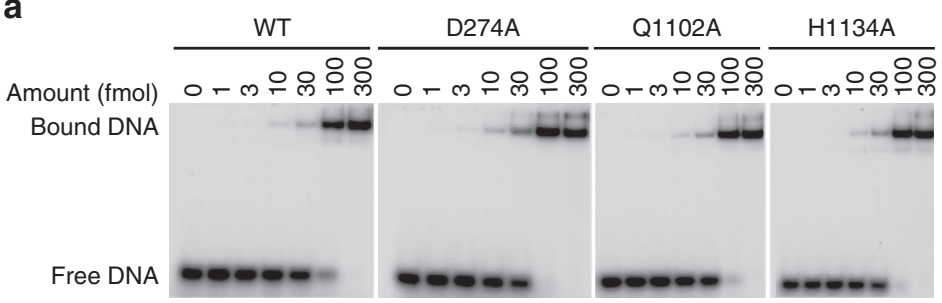

b

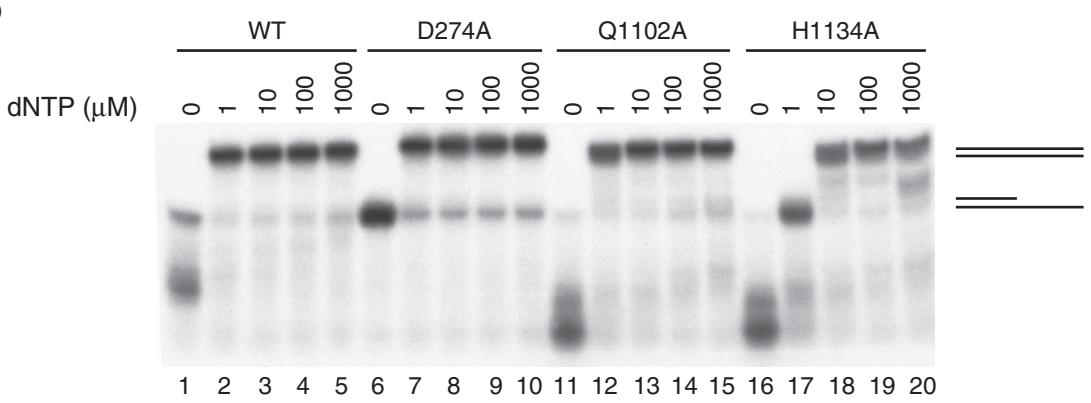

C

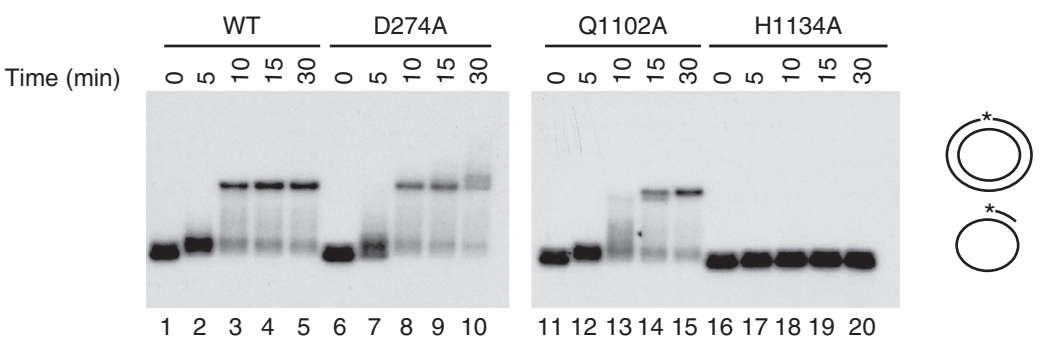

d

e
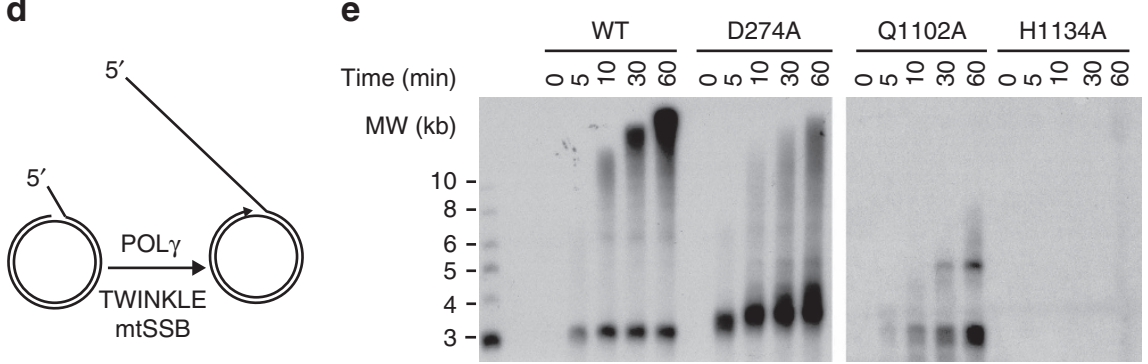

Figure 1 | In vitro characterization of HsPOL $\gamma$ A D274A, Q1102A and H1134A mutant proteins. (a) Representative gels showing electrophoretic mobility assays using WT and mutant $\mathrm{HsPOL} \gamma$ holoenzymes ( $\mathrm{HsPOL} \gamma \mathrm{A} / \mathrm{HsPOL} \gamma \mathrm{B}$ in complex) to estimate the Kd (DNA) values. Each lane contains 10 fmol of DNA substrate and the indicated amounts of protein complex. Values of dissociation constants presented as an average from three independent binding assays are shown on Table 1. (b) Coupled exonuclease-polymerase assay. Using the same template as in 'a' but in the presence of increasing amounts of dNTPs, H1134A required higher dNTP concentrations than the other HsPOL $\gamma$ A variants to polymerize the 35 -mer. Reactions were run on a denaturing $15 \%$ PAGE. (c) Second strand synthesis. Q1102A was a slower DNA polymerase than the WT and D274A proteins, whereas H1134A was not able to produce long stretches of DNA. The template consisted of a $5^{\prime}$ radioactively labelled (asterisk represents the labelling) 32-mer hybridized to a single-stranded pBluescript DNA. (d) Schematic representation of the rolling circle in vitro replication assay used to analyse the function of different $\mathrm{HsPOL} \gamma \mathrm{A}$ variants in the context of the minimal mitochondrial replisome. In the presence of mtSSB and the TWINKLE helicase, POL $\gamma$ is able to synthesize long stretches of DNA. (e) Rolling circle in vitro replication assay. The time curve shows the inability of the H1134A variant to produce long stretches of DNA also in the context of the replisome. Also Q1102A had also problems in generating long products. All experiments were repeated at least three times.

Human recombinant HsPOL $\gamma$ A proteins were purified to near homogeneity after expression in insect cells to obtain WT, D274A, Q1102A and H1134A variants (Supplementary Fig. 2a). Unfortunately, despite several attempts we were not able to purify stable and enzymatically active recombinant DmPOL $\gamma \mathrm{A}$. Therefore all of the in vitro experiments were performed using the recombinant human proteins. We first tested the ability of these recombinant HsPOL $\gamma \mathrm{A}$ proteins to bind DNA in the presence of the processivity factor HsPOL $\gamma \mathrm{B}$ by using a primertemplate and standard electrophoretic mobility shift assay (EMSA) (Supplementary Fig. 2b). All HsPOL $\gamma$ A mutants bound
DNA and interacted with HsPOL $\gamma \mathrm{B}$ in a manner indistinguishable from the WT enzyme (Supplementary Fig. 2b). We also determined the Kd for DNA binding for all of the HsPOL $\gamma$ mutants and found values that were very similar to the Kd for the WT enzyme (Fig. 1a and Table 1).

The WT HsPOL $\gamma$ A has an exonuclease activity and will engage in $3^{\prime}$ to $5^{\prime}$ degradation of DNA in the absence of deoxynucleotide (dNTP)s (Fig. 1b, lane 1), whereas it exerts polymerase activity in the presence of dNTPs (Fig. 1b, lanes 2-5). The recombinant D274A protein displayed severely reduced exonuclease activity (Fig. 1b, lane 6), while maintaining polymerase activity (Fig. 1b, 
lane 7-10). In contrast, the Q1102A and $\mathrm{H} 1134 \mathrm{~A}$ variants had more pronounced exonuclease activities (Fig. 1b, lanes 11 and 16) compared with the WT protein (Fig. 1b, lane 1), indicating increased fidelity ${ }^{44}$. While the Q1102A variant retained a polymerase activity (Fig. $1 \mathrm{~b}$, lanes $12-15$ ) similar to the WT enzyme on a short template (Fig. 1b, lanes 2-5), the H1134A mutant protein required higher dNTP concentrations to synthesize DNA (Fig. 1b, lanes 17-20). We additionally tested whether any of the HsPOL $\gamma \mathrm{A}$ mutant variants were capable of synthesizing long stretches of DNA using a circular ssDNA template. The D274A variant fully replicated circular ssDNA with a similar efficiency as the WT polymerase (Fig. 1c). The Q1102A variant was also able to synthesize a full-length product, albeit at a slower rate (Fig. 1c lanes 11-15), whereas the H1134A variant was unable to replicate DNA under these assay conditions (Fig. 1c, lanes 16-20). The low polymerase activity of the two $\mathrm{pol}^{-}$variants was also verified in a processivity assay and the defect was again more severe for the H1134A mutant than for the Q1102A mutant (Supplementary Fig. 2c). In competition assays, we found that the H1134A mutant had some dominant negative effects (Supplementary Fig. 2d). We also tested whether the HsPOL $\gamma$ A variants could initiate DNA replication in vitro by performing rolling circle replication assays (Fig. 1d) ${ }^{15}$. Both WT and D274A enzymes generated DNA products of $\sim 20 \mathrm{~kb}$ in the presence of the mitochondrial replicative helicase TWINKLE, although D274A was a bit less efficient (Fig. 1e). In contrast, the pol $^{-}$mutations Q1102A and H1134A failed to synthesize long DNA products (Fig. 1e). The in vitro DNA replication results thus show that the D274A variant of HsPOL $\gamma \mathrm{A}$ has reduced exonuclease and high polymerase activities, whereas the Q1102A and $\mathrm{H} 1134 \mathrm{~A}$ variants have increased exonuclease and reduced polymerase activities.

DmPOL $\gamma$ A (tamas) KO flies show developmental lethality. We next proceeded to generate a founder line to allow subsequent introduction of mutant alleles into the endogenous tamas locus by using genomic engineering. This strategy ensures that the expression of the mutant DmPOL $\gamma \mathrm{A}$ variants is regulated in a physiological way, which is important because previous studies have shown that forced expression of DmPOL $\gamma \mathrm{A}$ in flies results in mtDNA depletion and lethality likely caused by an imbalance in the levels of DmPOL $\gamma \mathrm{A}$ and DmPOL $\gamma \mathrm{B}^{47}$. To this end, we first generated DmPOL $\gamma \mathrm{A}$ KO flies by replacing the tamas gene with a short attP site after 'ends-out' homologous recombination (Supplementary Fig. $3 a)^{48}$. Precise excision of the tamas gene and insertion of the attP site was verified by PCR and sequencing (Fig. 2a and Supplementary Fig. 3b). The tamas KO larvae contained no detectable tamas mRNA whereas the mRNA expression from neighboring genes was normal (Fig. 2b). The $\mathrm{KO}$ of tamas was thus efficient and the presence of the attP sequence does not disturb the expression of the adjacent genes. Heterozygous DmPOL $\gamma \mathrm{A}$ KO flies $(\mathrm{KO} /+)$ developed normally to adulthood, whereas intercrosses produced no homozygous $\mathrm{KO}$

\section{Table 1 | The Kd (DNA) values of WT and mutant HsPOL $\gamma$} holoenzymes (HsPOL $\gamma$ A/HsPOL $\gamma$ B in complex).

\begin{tabular}{lc} 
Pol $\boldsymbol{\gamma}$ holoenzyme & DNA binding Kd (nM) \\
\hline WT & $5.5 \pm 1.4$ \\
D274A & $2.8 \pm 0.1$ \\
Q1102A & $3.8 \pm 0.2$ \\
H1134A & $3.5 \pm 0.5$
\end{tabular}

WT, wild type.

Table shows values of dissociation constants as an average from three independent binding assays with errors presented as s.d.
$(\mathrm{KO} / \mathrm{KO})$ flies, thus showing that DmPOL $\gamma \mathrm{A}$ is essential for fly survival. To show that the lethality phenotype is caused by loss of tamas, we performed genetic complementation tests between tamas KO mutants and deficiency lines that cover the locus (Supplementary Fig. 3d and Supplementary Table 1). The deficiencies that cover the tamas locus could not rescue the phenotype, whereas a deficiency adjacent to the tamas locus fully rescued the tamas KO mutant (Supplementary Table 1). The different deficiencies could not complement each other (Supplementary Table 1). The homozygous tamas KO larvae $(\mathrm{KO} / \mathrm{KO})$ died at the third instar (L3) stage (Fig. 2c) with a severe reduction in body weight (Supplementary Fig. 3c) and a $70 \%$ decrease in mtDNA levels (Fig. 2d). The residual mtDNA levels in the tamas $\mathrm{KO} / \mathrm{KO}$ larvae are most likely explained by maternal contribution of both mtDNA and DmPOL $\gamma \mathrm{A}$ to the embryo, which allows the homozygous KOs to develop until the L3 stage. A similar maternal contribution is well-documented in mammals, where mouse embryos with severe mtDNA replication defects survive until after implantation ${ }^{9,11,49}$, and in Caenorhabditis elegans, where loss of POL $\gamma$ leads to apparently normal development albeit with severely reduced gonadal function ${ }^{50}$.

Homozygous DmPOL $\gamma \mathrm{A}$ exo ${ }^{-}$and pol $^{-}$flies arrest in development. POL $\gamma \mathrm{A}$ is highly conserved (Supplementary Fig. 1) and the human and fly orthologues have $56.1 \%$ amino acid identity (http://mobyle.pasteur.fr). The residues D274, Q1102 and H1134 of HsPOL $\gamma \mathrm{A}$ correspond to residues D263, Q1009 and H1038 of DmPOL $\gamma$ A, respectively (Supplementary Fig. 1). We utilized site-specific integration and the attP site ${ }^{48}$ to introduce mutant alleles into the tamas locus (Supplementary Fig. 3a) and generated flies expressing the D263A, Q1009A or H1038A variants of DmPOL $\gamma$ A (Supplementary Fig. 4a-d). For simplicity, we hereafter refer to the corresponding alleles as the D263A, Q1009A and H1038A alleles, respectively. As a control, we also introduced WT DmPOL $\gamma \mathrm{A}$ to rescue the KO allele. Correct re-integration of the introduced tamas alleles was confirmed by Southern blot and PCR analyses (Fig. 3a, Supplementary Fig. 4a). Reintroduction of WT DmPOL $\gamma \mathrm{A}$ (rescue allele) resulted in apparently normal homozygous flies, which demonstrate that the lethality of tamas $\mathrm{KO}$ flies is caused by the absence of DmPOL $\gamma \mathrm{A}$ (Fig. 3b). In addition, the rescue allele had no or very mild impact on the expression of neighbouring genes in 5-day-old rescue larvae (L3 stage), thus excluding positional effects in the targeted region (Supplementary Fig. 4c). Larvae homozygous for the D263A, Q1009A or H1038A alleles died mostly at the late L3 stage (Supplementary Fig. 4d and Supplementary Table 2) although some D263A larvae entered the pupal stage and died shortly thereafter (Supplementary Table 4). Body size and weight of homozygous rescue or D263A larvae were comparable to WT larvae, while H1038A and Q1009A mutants were significantly smaller (Fig. 3c and Supplementary Fig. 4d). Furthermore, excision of the mini-white marker (Supplementary Fig. 4b) did not affect the survival or visible phenotypes of the genomically engineered flies, which shows that the observed phenotypes are a direct consequence of the introduced mutant variants of DmPOL $\gamma$ A.

The oxygen consumption rates in permeabilized tissues were normal in 5-day-old homozygous rescue larvae (Fig. 3d). The homozygous D263A larvae did not display any detectable changes in state 3 or state 4 respiration (Fig. $3 \mathrm{~d}$ ), whereas the homozygous Q1009A or H1038A larvae showed a profound reduction in both state 3 and uncoupled respiration (Fig. 3d).

The rescue, D263A, Q1009A and H1038A alleles were crossed over the deficiencies described above as well as over the tam3 and tam4 hypomorphic POL $\gamma A$ alleles ${ }^{51}$. We only obtained 
a
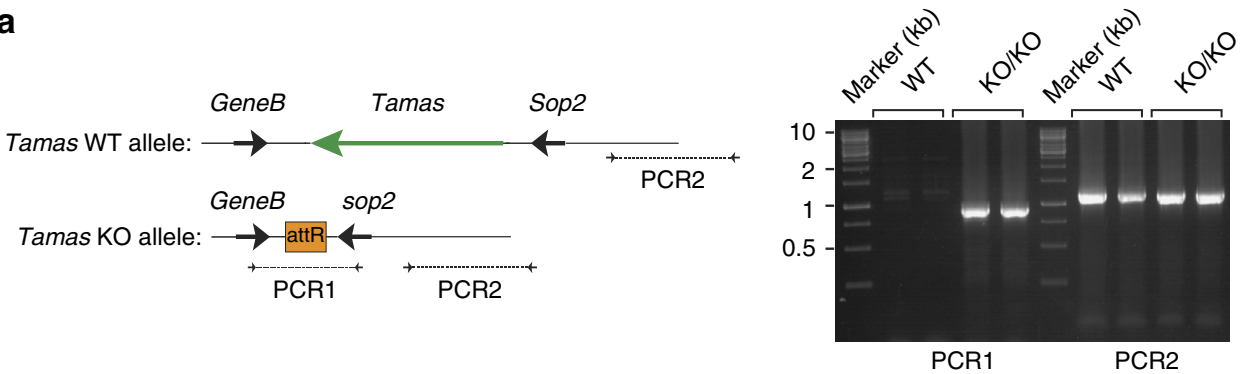

b

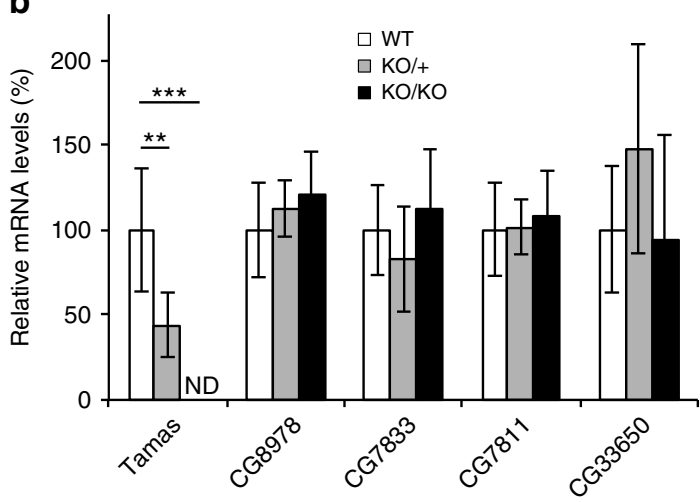

C
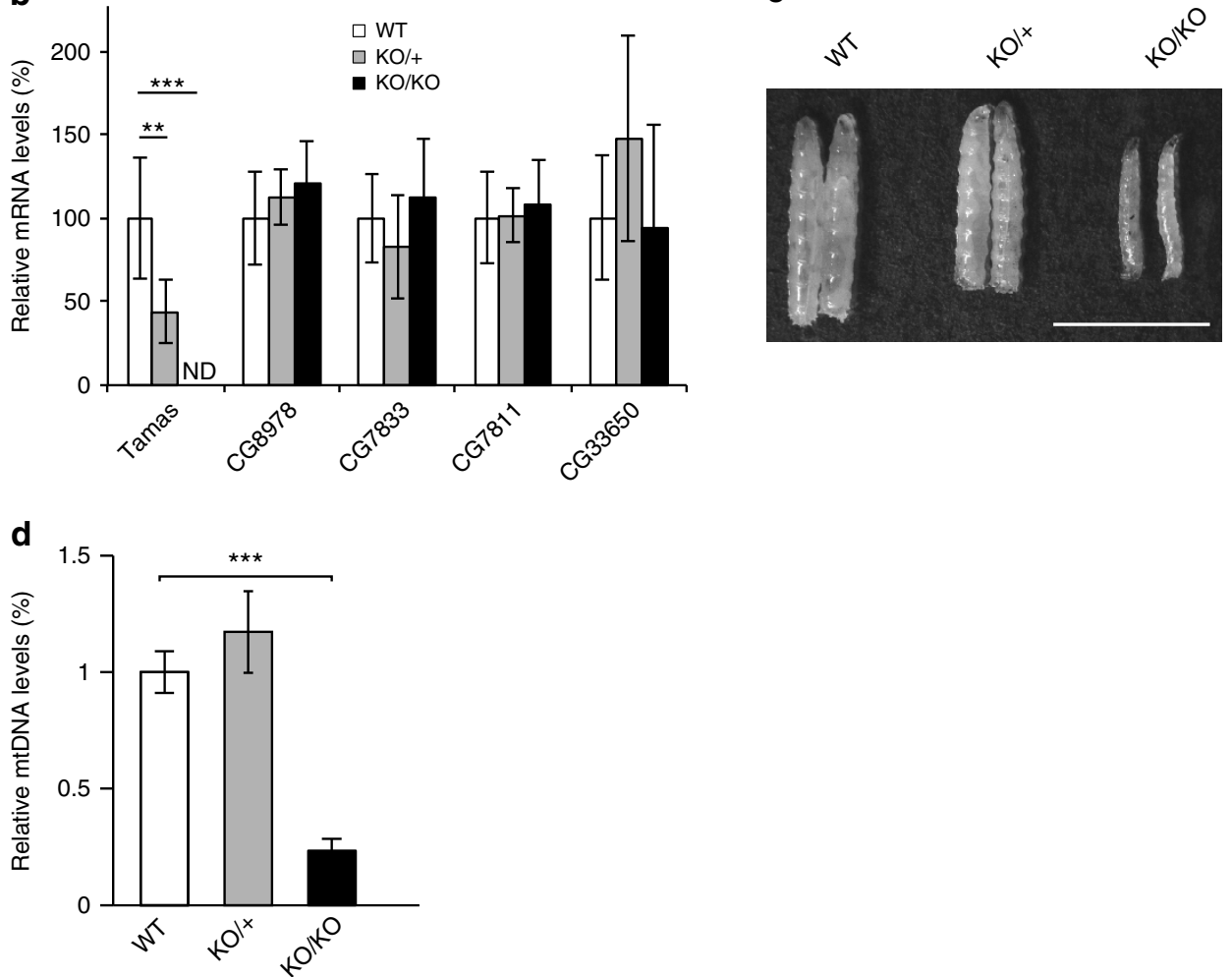

Figure 2 | Creation of a DmPOL $\gamma \mathbf{A}$ knockout founder line by using genomic engineering. (a) The $D m P O L \gamma A$ (tamas) locus and generation of $D m P O L \gamma A$ knockout (KO) mutant. Tamas gene encoding DmPOL $\gamma \mathrm{A}$ is located on the second chromosome at cytological position 34D1. The DmPOL $\gamma A$ knockout founder fly line was generated by ends-out homologous recombination. Deletion of the tamas gene was confirmed by PCR1, while PCR2 was used as a control. Total DNA was extracted from DmPOL $\gamma A$ knockout larvae (KO) and wild-type (WT) flies. (b) RNA expression levels of tamas and the flanking genes in the tamas knockout larvae (KO). The expression of DmPOL $\gamma \mathrm{A}$ (tamas) gene was not detected (ND) in the homozygous (KO/KO, black bar) knockout larvae. The expression of DmPOL $\gamma$ A (tamas) neighbouring genes was not affected by removal of tamas allele in DmPOL $\gamma A$ heterozygous (KO/ + , grey bar) and homozygous (KO/KO, black bar) knockout larvae. Total RNA was extracted from 5-day-old larvae and gene expression was analysed by qRT-PCR. One-way ANOVA with Dunnett's post hoc test. ${ }^{\star \star} P<0.001$, ${ }^{\star \star} P<0.01$. Error bars represent s.d. $n=5$. Data represent two independent experiments. (c) Comparison of body size between wild-type (WT, white bar), heterozygous (KO/ +, grey bar) and homozygous DmPOL $\gamma A$ knockout (KO/KO, black bar) larvae. Homozygous knockout larvae were smaller than the wild-type and heterozygous knockout larvae. Scale bar, 5 mm.

(d) Steady-state levels of mtDNA were determined by quantitative PCR in 5-day-old wild-type (WT, white bar), heterozygous (KO/ + , grey bar) and homozygous (KO/KO, black bar) DmPOL $\gamma A$ knockout larvae. Lack of DmPOL $\gamma A$ led to severe mtDNA depletion. Data represent at least three independent experiments. Kruskal-Wallis test with Dunnett's post hoc test. ${ }^{\star \star \star} P<0.001$. Error bars represent s.d. $n=4-6$.

complementation with the rescue allele and it complemented all of the deficiencies covering the tamas locus, as well as the tam3 and tam4 hypomorphic alleles (Supplementary Table 3). Developmental assays were performed with the exo- ${ }^{-}$and $\mathrm{pol}^{-}$ tamas alleles crossed over the tamas $\mathrm{KO}$ allele to detect any possible antimorphic effects (Supplementary Table 4). The results were strongly dependent on whether the mutant allele was transmitted maternally or paternally, and we therefore performed the crosses in both directions. In all studied combinations, the homozygous tamas $\mathrm{KO}$ allele had the most severe developmental lethality phenotype (Supplementary Table 4). The homozygous
$\mathrm{D} 263 \mathrm{~A} \mathrm{exo}^{-}$flies died in the early pupal stage, whereas hemizygous flies with paternal (KO/D263A) or maternal (D263A/KO) transmission of the D263A allele generated sick escapers that reached the adult stage. The homozygous $\mathrm{pol}^{-}$flies died before pupariation, whereas the hemizygous $\mathrm{pol}^{-}$flies with a paternally transmitted mutant allele $(\mathrm{KO} / \mathrm{H} 1038 \mathrm{~A}$ and $\mathrm{KO} / \mathrm{Q} 1009 \mathrm{~A})$ reached the pupal stage. The larval body weight (Supplementary Fig. 5a) and the mtDNA levels (Supplementary Fig. 5b) correlated with the developmental phenotype. However, the H1038A allele showed a different pattern as these larvae had the same low body mass as the KO larvae, but less mtDNA than 

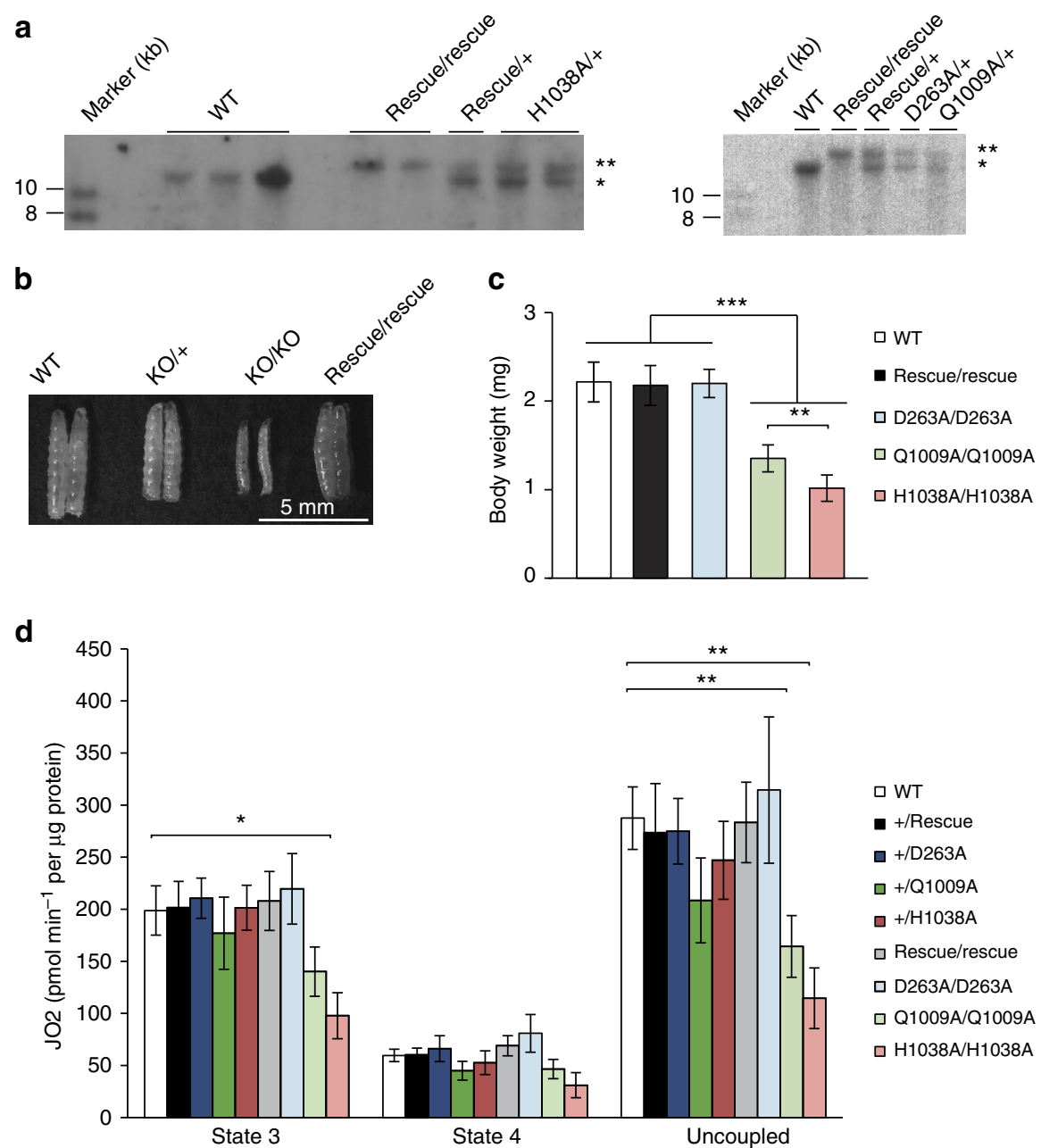

Figure 3 | Phenotypical characterization of the genomically engineered DmPOL $\gamma \mathbf{A}$ exo ${ }^{-}$and pol $^{-}$flies. (a) Verification of precise re-integration of tamas allelic variants by Southern blot analysis. Restriction of genomic DNA with Xhol generated a $\sim 12.1 \mathrm{~kb}$ band in wild-type allele (one asterisk) and

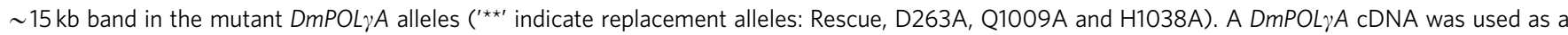
probe. Total DNA was extracted from adult flies. (b) Comparison of body size between wild-type, heterozygous $(\mathrm{KO} /+)$ and homozygous $\mathrm{DmPO}(\gamma \mathrm{A}$ knockout $(K O / K O)$ and homozygous rescue larvae. Re-introduction of the WT allele (rescue) to the genomically engineered $D m P O L \gamma A$ locus rescued the phenotypes of the knockout larvae. Scale bar, $5 \mathrm{~mm}$. (c) Quantification of the body weight of genomically engineered DmPOL $\gamma A$ homozygous larvae. Homozygous DmPOL $\gamma$ A Q1009A and H1038A larvae were significantly smaller than the wild-type (WT), rescue and D263A larvae. Tukey's Multiple Comparison Test. ${ }^{\star \star \star} P<0.001,{ }^{\star \star} P<0.01$. Error bars represent s.d. $n=20$. All indicated genotypes correspond to 5-day-old homozygous larvae. (d) Respiratory chain function. Oxygen consumption rates of 5-day-old larvae were measured under phosphorylating (state 3), non-phosphorylating (state 4) and uncoupled conditions and normalized to total protein content. Data represent two to three independent experiments. Mann-Whitney test, two-tailed. ${ }^{\star \star} P<0.01,{ }^{\star} P<0.05$. Error bars represent s.d. $n=4$.

KO larvae (Supplementary Fig. 5a,b). The H1038A mutant thus had some dominant negative effects, which is consistent with the in vitro biochemistry results of the corresponding HsPOL $\gamma \mathrm{A}$ mutant (H1134A; Supplementary Fig. 2d).

DmPOL $\gamma$ exo ${ }^{-}$mainly introduces transition mutations of mtDNA. We performed extensive cloning and sequencing to quantify the mtDNA mutation loads in the three mutated fly lines, as well as in the rescue line. The mtDNA mutation levels were low in WT flies $\left(\sim 10^{-5}\right.$ mutations per bp), consistent with a previous report ${ }^{24}$. In contrast, the heterozygous D263A mutant flies had significantly increased mtDNA mutation levels with on an average $7 \times 10^{-5}$ mutations per bp (Fig. 4). In 5-day-old larvae homozygous for the D263A mutant allele (mtDNA mutator larvae), a further increase of the mtDNA mutation load $\left(\sim 2 \times 10^{-4}\right.$ mutations per bp) was observed (Fig. 4). The heterozygous D263A mutant flies carried more transition mutations (purine $>$ purine or pyrimidine $>$ pyrimidine) than transversions (purine $>$ pyrimidine or pyrimidine $>$ purine), and all possible transitions occurred at an equal frequency (Table 2). In contrast, the $\mathrm{T}>\mathrm{A}$ transversions (relative to the reference sequence) were much more frequent than the sum of all other transversion mutations (Table 2). Heterozygous flies carrying the Q1009A or H1038A high-fidelity alleles had mtDNA point mutation loads similar to the controls (Fig. 4).

Linear mtDNA deletions in homozygous DmPOL $\gamma \mathrm{A}$ exo- ${ }^{-}$larvae. We performed quantitative PCR (qPCR) and Southern blot analyses of larvae heterozygous for the D263A, Q1009A and H1038A alleles and found no significant changes in mtDNA steady-state levels in comparison with WT larvae (Fig. 5a). Larvae homozygous for the D263A allele displayed a mild reduction in mtDNA levels (Fig. 5a), whereas larvae homozygous for the Q1009A and H1038A alleles showed severe mtDNA depletion 


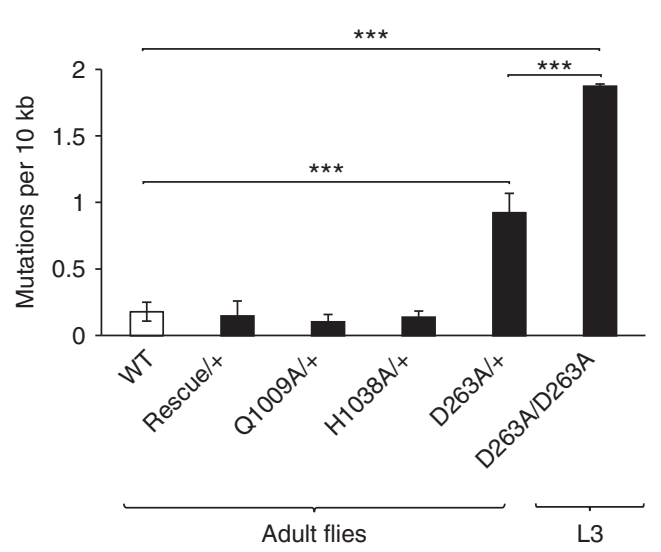

Figure 4 | Quantitative assessment of mtDNA mutations in larvae and flies carrying different $\operatorname{DmPOL} \gamma \boldsymbol{A}$ alleles. Post-PCR cloning and sequencing was used to quantify mtDNA mutation load in young adult wild-type (WT), genomically engineered heterozygous DMPOL $\gamma A$ flies (Rescue/ +, Q1009A/ +, H1038A/ +, D263A/ + ) and homozygous exo- larvae (D263A/D263A). If no mutations were detected, the error rate of our method (3.5E - 06) was used for further analysis. Bars represent the average number of unique mtDNA mutations. All flies inherited the mutated DmPOL $\gamma$ A allele maternally, hence the detected mIDNA mutations represent both inherited and somatic mutations. One-way ANOVA with Tukey's test. ${ }^{\star \star} P<0.001$. Error bars represent s.d. $n=3-4$.

(Fig. 5a), which is consistent with the in vitro biochemistry results (Fig. 1, Supplementary Fig. 2c,d).

Interestingly, Southern blot analysis revealed the presence of high levels of two species of linear mtDNA molecules with deletions in larvae homozygous for the D263A allele (Fig. 5b,c). These aberrant molecules were not observed in the heterozygous D263A larvae (Supplementary Fig. 6a). Detailed mapping of the linear mtDNAs with deletions revealed two species that differed by $\sim 1 \mathrm{~kb}$ in size (Fig. $5 \mathrm{c}$ ). The ends of the linear mtDNAs with deletions reach the $\mathrm{A}+\mathrm{T}$-rich control region, which have been suggested to contain both the $\mathrm{O}_{\mathrm{H}}$ and $\mathrm{O}_{\mathrm{L}}$ for fly mtDNA $(\text { Fig. } 5 b)^{52}$. The linear mtDNA with deletions already appears in the second instar stage ( 3 days after egg laying (AEL)), and their levels reach $\sim 40 \%$ of total mtDNA at 5 days AEL (Fig. $5 \mathrm{~d}$, Supplementary Fig. 6b), meaning that the homozygous D263A mutant larvae have a deficiency of full-length mtDNA (Fig. 5d). Southern blot analysis of the polymerase domain mutants, Q1009A and H1038A revealed no deletions (Supplementary Fig. 6c), demonstrating that the exonuclease-deficient DmPOL $\gamma \mathrm{A}$ is directly involved in generating the linear mtDNA deletions.

To determine whether the homozygous D263A larvae are dying because of depletion of full-length mtDNA molecules, we determined the amount of mtDNA needed to enter and go through the pupariation stage. To this end, we characterized, molecularly and phenotypically, two independent TFAM knockdown lines, as TFAM levels are known to control mtDNA copy number. Our results show that most TFAM RNAi flies enter the pharate stage despite having a lower mtDNA copy number than homozygous D263A larvae (Fig. 5e and Supplementary Table 5). This shows that the mtDNA depletion in the homozygous D263A larvae is not sufficient on its own to cause the observed lethality.

DmPOL $\gamma \mathrm{A} \mathrm{exo}^{-}$flies slowly accumulate mtDNA mutations. We first studied how mutation levels change during development in flies that have only somatic mutagenesis of mtDNA, that is, flies that are heterozygous for a paternally transmitted D263A

\begin{tabular}{|c|c|c|c|}
\hline Mutation & & Fractior & \\
\hline \multicolumn{4}{|l|}{ Transition } \\
\hline$A>G$ & 0.13 & 0.25 & 0.51 \\
\hline $\mathrm{T}>\mathrm{C}$ & 0.12 & & \\
\hline$C>T$ & 0.15 & 0.26 & \\
\hline$G>A$ & 0.12 & & \\
\hline \multicolumn{4}{|c|}{ Transversion } \\
\hline$A>C$ & 0.00 & 0.02 & 0.25 \\
\hline $\mathrm{T}>\mathrm{G}$ & 0.02 & & \\
\hline$A>T$ & 0.04 & 0.18 & \\
\hline$T>A$ & 0.14 & & \\
\hline$C>A$ & 0.01 & 0.05 & \\
\hline$G>T$ & 0.04 & & \\
\hline$C>G$ & 0.00 & 0.00 & \\
\hline$G>C$ & 0.00 & & \\
\hline Indel & & 0.24 & \\
\hline \multicolumn{4}{|c|}{$\begin{array}{l}\text { Data were pooled from a total number of } 18 \text { flies. In the first column different mutation } \\
\text { frequencies are shown from the perspective of the heavy strand. In our method it is not possible } \\
\text { to distinguish if the mutation has originally taken place in the heavy strand (that is, AG) or in the } \\
\text { light strand (TC). Therefore the frequencies of these indistinguishable mutations have been } \\
\text { combined in the second column. In the third column, the frequencies of transitions, } \\
\text { transversions and indel mutations are compared showing that most of the detected mutations } \\
\text { are transitions (51\%). }\end{array}$} \\
\hline
\end{tabular}

allele (Fig. 6a). Interestingly, there was a burst in mtDNA mutation levels after morphogenesis (Fig. 6a). Next we proceeded to study how these mtDNA mutations are transmitted between generations. To this end, we first crossed heterozygous D263A male flies to WT females and measured the mtDNA mutation load in the offspring (Fig. 6b). As the analysed flies were born to mothers that only had transmitted WT mtDNA, maternally transmitted mtDNA mutations cannot influence the overall mtDNA mutation levels. This cross thus allowed us to selectively determine the somatic mtDNA mutation load generated by the heterozygous D263A allele (Fig. 6b). Next, we performed crosses with female flies that had inherited their mtDNA from a maternal lineage of flies heterozygous for the D263A allele for 1, 4, 6 or 13-15 generations (Fig. 6b). Surprisingly, we observed that the accumulation of mtDNA mutations between generations occurred very slowly in flies (Fig. 6b). In fact, the mtDNA mutation load in flies heterozygous for the D263A allele was very similar in strains with reintroduced WT mtDNA and in strains intercrossed for up to four generations (Fig. 6b). However, on further intercrossing, for six generations or more, increased mtDNA mutation levels were observed (Fig. 6b) and the total mutation levels were comparable to those of homozygous D263A larvae (Fig. 4). Altogether, these observations show that flies can maintain a wider mtDNA sequence variation between generations than mammals ${ }^{41}$. It is generally accepted that a small genetic bottleneck in the mammalian maternal germline cause rapid shifts in mtDNA genotypes between generations ${ }^{41}$. Therefore, the slow accumulation of mtDNA mutations between generations in heterozygous D263A flies suggests that there is a wider size of the genetic bottleneck for maternal transmission of mtDNA in flies than in mammals.

The DmPOL $\gamma \mathrm{A}$ exo ${ }^{-}$and pol $^{-}$variants can complement in trans. The finding that flies that are heterozygous for the D263A allele have about half of the mtDNA mutation load of larvae that are homozygous for the D263A allele suggest that the WT allele can complement in trans. We performed a series of crosses to obtain compound heterozygous flies to investigate the occurrence of genetic complementation (Supplementary Fig. 7a). The two pol $^{-}$mutants Q1009A and H1038A could not complement each 
a

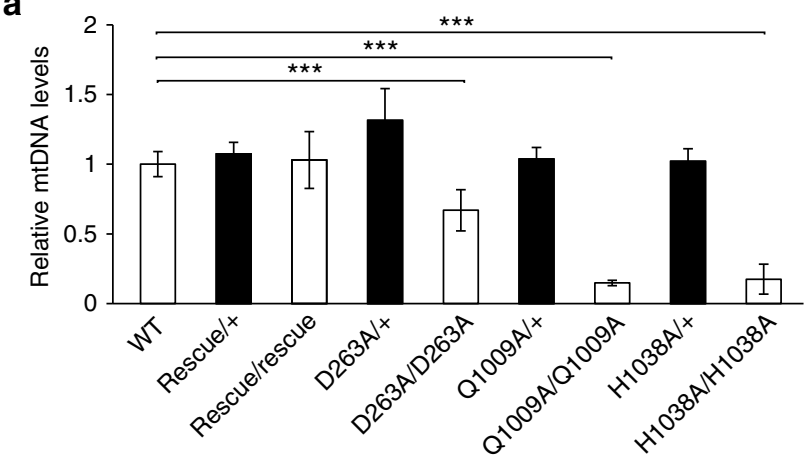

$\square$ Homozygous

- Heterozygous

b

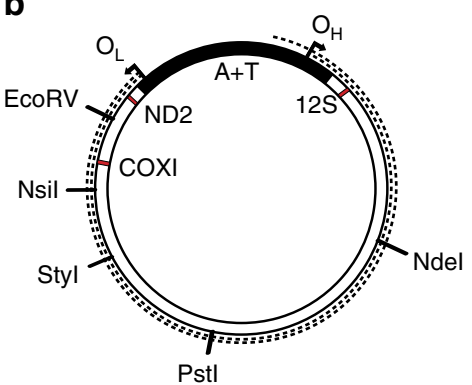

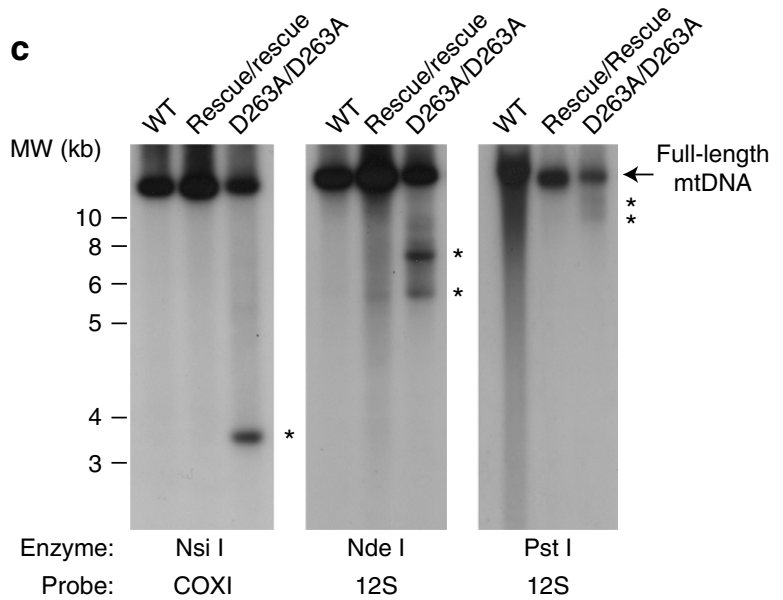

d

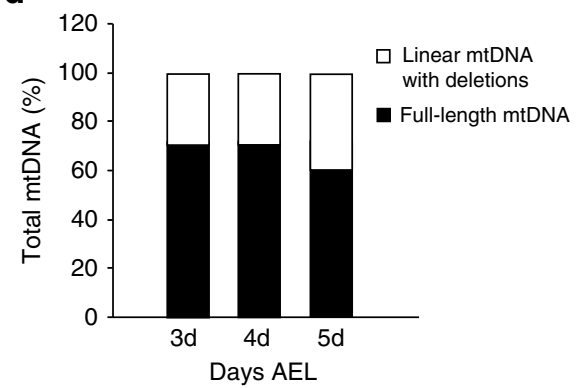

e

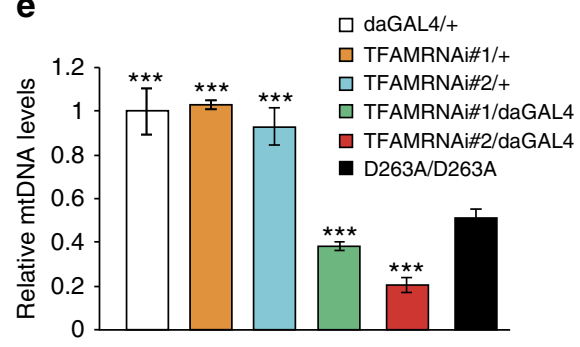

Figure 5 | Quantification of mtDNA copy number and analysis of mtDNA integrity. (a) Quantification of mtDNA levels in genomically engineered DmPOL $\gamma$ A flies. Steady-state levels of mtDNA were determined by quantitative PCR in 5-day-old homozygous (white bar) and heterozygous (black bar) larvae. Data represent two to four independent experiments. Kruskal-Wallis test with Dunnett's post hoc test. ${ }^{\star \star \star} P<0.001$. Error bars represent s.d. $n=4-6$. (b) Schematic representation of Drosophila mtDNA. The dotted lines indicate linear mtDNA molecules with deletions. Restriction endonucleases used for mapping the deletions in the mtDNA are indicated. Red bars represent oligonucleotide probes used to map the mtDNA deletions. $\mathrm{O}_{H}$ (origin of replication for heavy strand of $m t D N A$ ) and $O_{L}$ (origin of replication for light strand of $m t D N A$ ), $A+T$ (control region of fly mtDNA). (c) Mapping mtDNA deletions. MtDNA was cut with Nsil (left panel), Ndel (middle panel) or Pstl (right panel). MtDNA deletions were mapped with COXI and 125 rRNA probes. (d) The relative amount of mtDNA deletions increases throughout the larval development in homozygous DmPOL $\gamma A$ D263A mutants. Total DNA was extracted from 3-day-, 4-day- and 5-day-old homozygous D263A exo- larvae and restricted with Styl. MtDNA deletions were detected using a COXI probe. Data represent two independent experiments. (e) Ubiquitous TFAM knockdown leads to severe mtDNA depletion. TFAM knockdown lead to a more pronounced decrease in mtDNA copy number in comparison with homozygous exo- larvae. All genotypes were compared against the homozygous exo- larvae (D263A/D263A). Data represent two independent experiments. One-way ANOVA with Dunnett's post hoc test. ${ }^{\star \star \star} P<0.001$. Error bars represent s.d. $n=5$.

other to generate viable compound heterozygous flies (Supplementary Table 2). However, compound heterozygotes, expressing either of the $\mathrm{pol}^{-}$mutants from one allele and the exo $^{-}$mutant from the other allele, were viable and apparently normal (Fig. 7a and Supplementary Fig. 7b-d and Supplementary Table 2). We observed no differences in body weight (Fig. 7a) or oxygen consumption rates (Supplementary Fig. $7 \mathrm{~b}$ ) of these compound heterozygous larvae in comparison with controls, irrespective of whether the D263A allele was paternally (Q1009A/ D263A and H1038A/D263A) or maternally (D263A/Q1009A and D263A/H1038A) inherited. Furthermore, we found no differences in total mtDNA levels between compound heterozygous and WT larvae (Supplementary Fig. 7c) and no linear mtDNAs with deletions were present (Supplementary Fig. 7d). Based on these findings, we conclude that compound heterozygotes harbouring one exo ${ }^{-}$(D263A) and one $\mathrm{pol}^{-}$allele (Q1009A or 

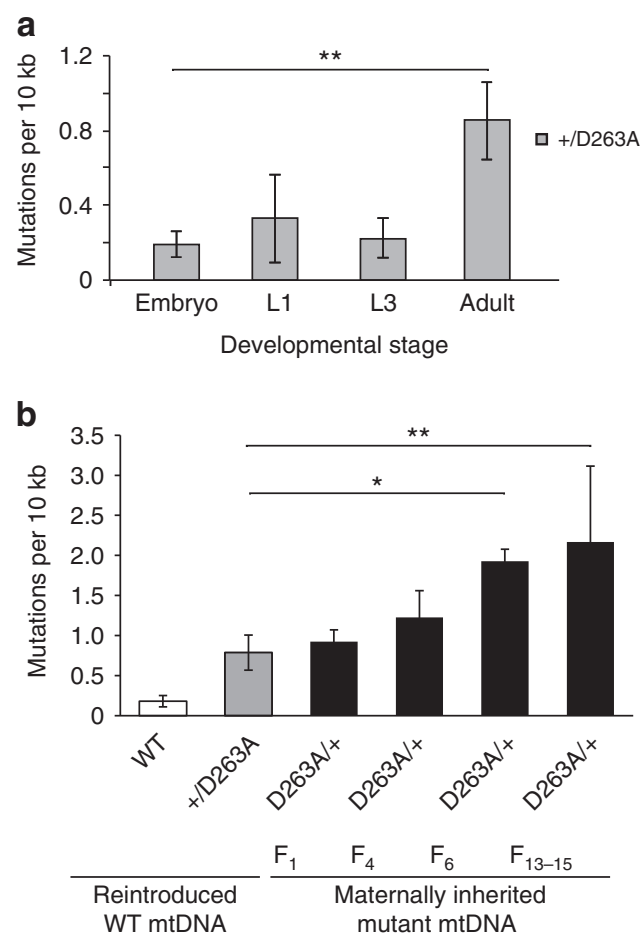

Figure 6 | MtDNA mutations increase after morphogenesis and accumulate transgenerationally. (a) MtDNA mutation load was determined in heterozygous D263A flies in different developmental stages. The D263A exo- allele was transmitted paternally and therefore all the detected mutations are produced by somatic mutagenesis. An increase in the mtDNA mutation load relative to wild-type (WT) flies was only detected after morphogenesis. One-way ANOVA with Dunnett's post hoc test. ${ }^{\star \star} P<0$.01. Error bars represent s.d. $n=3-6$. (b) MtDNA mutations accumulated in heterozygous D263A exo- flies after successive intercrossing for several generations. MtDNA mutation loads were compared among wild-type flies (WT, white bar), heterozygous D263A exo $^{-}$flies with a clean background (+/D263A, lack maternally transmitted mtDNA mutations, grey bar) and heterozygous D263A exo- flies that maternally inherited mtDNA mutations for 1, 4, 6 or 13-15 generations (D263A/ +, black bar). One-way ANOVA with Dunnett's post hoc test. ${ }^{\star}{ }^{\star} P<0.01,{ }^{\star} P<0.05$. Error bars represent s.d. $n=3-6$.

H1038A) are phenotypically normal due to genetic complementation.

Complementing flies have clonally expanded mtDNA mutations. We proceeded to investigate the mtDNA mutation load in larvae to get insights into why compound heterozygous (D263A/ H1038A) but not homozygous exo ${ }^{-}$(D263A/D263A) larvae can proceed normally through development. There is accumulation of mtDNA mutations after morphogenesis (Fig. 6a) and any meaningful comparison of the mutation load must therefore be performed in larvae at the same developmental stage. The D263A/H1038A larvae had a similar total mutation load as the D263A/D263A larvae at the L3 stage (Supplementary Fig. 7e), but the number of unique mtDNA mutations was significantly lower (Fig. 7b). The high unique mtDNA mutation load and the presence of linear mtDNA deletions in D263A/D263A larvae likely explain why they cannot proceed through development.

Next, we studied adult flies that were compound heterozygous or only heterozygous for the D263A allele and found similar mutation loads if the D263A allele was paternally transmitted (Fig. 7c). In contrast, compound heterozygous flies containing a
D263A allele that had been maternally transmitted for one generation had a higher mtDNA mutation load (Fig. 7c). We maintained heterozygous D263A mutant flies by intercrosses for four generations and then crossed the obtained D263A females with H1038A males to generate compound heterozygotes (D263A/H1038A) (Supplementary Fig. 7a). Very unexpectedly, we observed a substantial increase in the total mtDNA mutation load in the compound heterozygous adult flies, which is mainly explained by high levels of clonally expanded mutations (Supplementary Fig. 7f), thus showing that the H1038A allele likely decreases the size of the bottleneck in the maternal germline. It should be noted that mtDNA mutations that have passed through the germline are subject to purifying selection $^{29,31,39}$ and therefore are less deleterious than mtDNA mutations that are somatically generated, which likely explains why the compound heterozygous flies are viable despite having high total mtDNA mutation loads.

Maternal mtDNA mutations cause developmental delay. We investigated the timing of development in different types of compound heterozygous flies, where the females contained re-introduced WT mtDNA, and in all cases the majority of flies eclosed at $\sim 11$ days AEL (Fig. $7 \mathrm{~d}$, left panel). However, the presence of the D263A allele in the mother led to a slight delay of eclosion in a subpopulation of flies (Fig. 7d, left panel), consistent with some germline mutagenesis of the re-introduced mtDNA. Next, we maintained heterozygous D263A mutant flies by intercrosses for four generations and then crossed them to obtain compound heterozygotes (Fig. 7d, right panel). Interestingly, significant developmental delay was observed in the compound heterozygous flies coming from maternal lineages where mtDNA mutations could accumulate (D263A/Rescue, D263A/H1038A and D263A/Q1009A; Fig. 7d, right panel). To exclude the possibility that acquired nuclear mutations could explain the observed developmental delays, we performed outcrosses to WT flies for two generations. Consistent with a maternal effect, we observed significant developmental delay only in the WT progeny with inherited mtDNA mutations (Fig. 7e). In this experiment, we also included heterozygous D263A flies that had been maintained by intercrosses for $\sim 2$ years and outcrossed to WT flies for six generations (Fig. 7e, green and red lines). A very significant developmental delay was observed in the outcrossed flies that were maternally related to the original cross (Fig. 7e, green line), whereas paternally related flies had normal timing of development (Fig. 7e, red line).

HsPOLyA exo ${ }^{-}$and pol $^{-}$proteins cooperatively replicate mtDNA. To investigate whether we could reconstitute the observed genetic complementation biochemically with recombinant mutant forms of HsPOL $\gamma \mathrm{A}$, we assessed elongation of a DNA primer containing a $3^{\prime}$ mismatch (Fig. 8a). The WT HsPOL $\gamma$ A could remove the $3^{\prime}$ mismatch by its exonucleolytic activity and then proceed to elongate in an efficient manner, whereas the D274A mutant could not efficiently remove the mismatch and switch to elongation (Fig. 8b). In contrast, both the Q1102A and H1134A mutants displayed increased exonuclease activity (Fig. 8b) in comparison with WT HsPOL $\gamma$ A (Fig. 8b). The Q1102A mutant was efficient in elongation, whereas the H1134A mutant was inefficient (Fig. 8b), which is in agreement with the severely reduced polymerase activity of the H1134A mutant (Fig. 1e). Finally, we performed in vitro complementation assays by mixing the D274A mutant with either Q1102A or H1134A mutants, using a replication primer that carried a $3^{\prime}$ mismatch nucleotide. On their own, neither the D274A nor the H1134A mutant was able to synthesize DNA efficiently (Fig. 8c, 
a

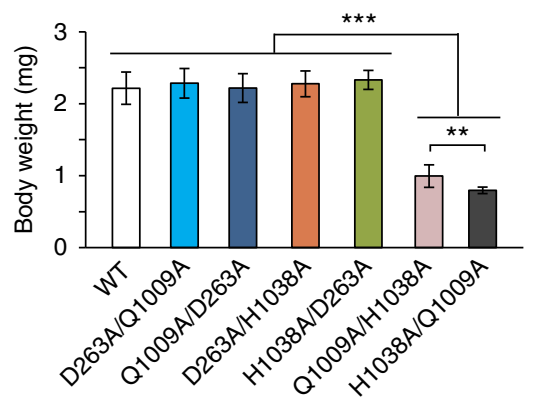

c
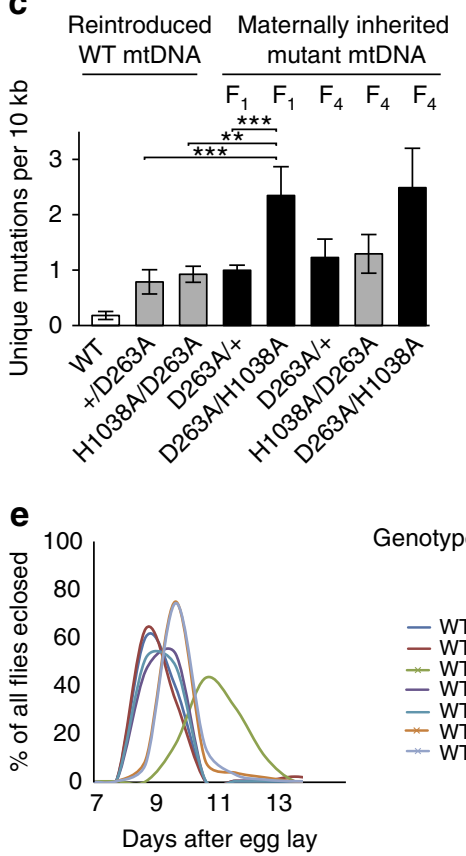

Genotype b

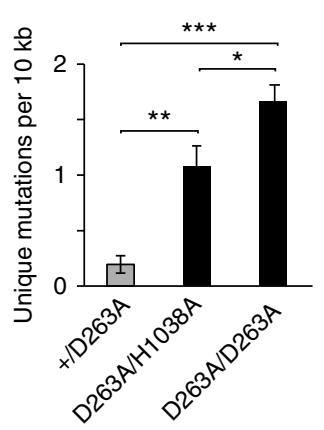

d

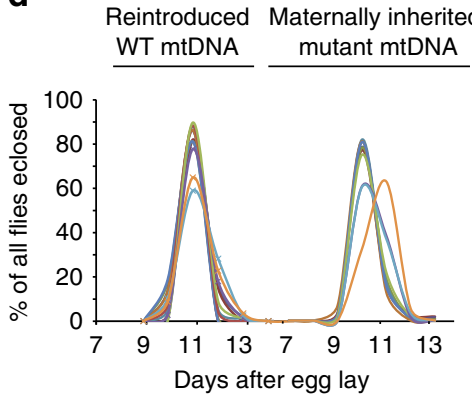

— WT

二 Rescue/GFP

- Rescue/D263A

- Rescue/Q1009A

- Rescue/H1038A

- Q1009A/rescue

- Q1009A/D263A

- H1038A/rescue

- H1038A/D263A

* D263A/Rescue

* D263A/Q1009A

$*$ D263A/H1038A

Parental

genotype

Inherited

mutant mtDNA

$\begin{array}{lll}\text { - WT } & \text { WT } & - \\ \text { - WT } & \text { D263A/+ } & - \\ \text { * WT } & \text { D263A/+ } & + \\ \text { - WT } & \text { Q1009A/D263A } & - \\ \text { - WT } & \text { H1038A/D263A } & - \\ \text { - WT } & \text { Q1009A/D263A } & + \\ * \text { WT } & \text { H1038A/D263A } & +\end{array}$

Figure 7 | Genetic complementation at the tamas locus. (a) Comparison of body weight between different $D m P O L \gamma A$ compound-heterozygous larvae. All DmPOL $\gamma$ A compound heterozygous larvae had wild-type-like body size. Body weight of 20 larvae (5-day old) was measured and average body weight is shown. Tukey's Multiple Comparison Test. ${ }^{\star \star}{ }^{\star} P<0.001,{ }^{\star \star} P<0.01$. Error bars represent s.d. $n=20$. (b) Quantification of unique mtDNA mutations in compound heterozygous larvae. The homozygous exo ${ }^{-}$larvae had significantly more unique mtDNA mutations in comparison with compound heterozygous larvae. Both genotypes inherited mtDNA mutations maternally for one generation. Tukey's Multiple Comparison test. ${ }^{\star \star \star} P<0.001,{ }^{\star \star} P<0.01$, ${ }^{\star} P<0.05$. Error bars represent s.d. $n=3$. (c) Quantification of unique mtDNA mutations in adult compound heterozygous flies without and with maternally transmitted mutations. Compound heterozygous flies with maternally transmitted D263A exo- allele (black bar) showed increase in the number of unique mtDNA mutations as compared with compound heterozygous flies that inherited the exo- ${ }^{-}$allele paternally (grey bar). One-way ANOVA with Dunnett's post hoc test. ${ }^{\star \star \star} P<0.001,{ }^{\star \star} P<0.01$. Error bars represent s.d. $n=3-6$. (d,e) An increase in the mtDNA mutation load affects fly development.

Developmental time of different complementation groups is shown. (d) All genomically engineered flies with a clean background (lacking maternally transmitted mtDNA mutations, left panel) had the same developmental time as wild-type (WT) flies. In contrast, all flies inheriting mtDNA mutations maternally for four generations (D263A/Rescue, D263A/H1038A and D263A/Q1009A) showed developmental delay (right panel). Crossing schemes are shown on Supplementary Fig. 7a. Data represent two independent experiments. (e) Wild-type (WT) flies carrying mtDNA mutations showed a severe developmental delay. Compound heterozygous flies used in $\mathbf{d}$ were outcrossed twice to replace tamas mutant alleles with wild-type tamas allele and clean the nuclear background. All flies had a WT nuclear background with or without maternally inherited mtDNA mutations.

lanes 4 and 8). However, when we mixed the two mutant proteins, they complemented each other and supported efficient DNA synthesis (Fig. 8c, lane 14) suggesting cooperation at the mismatched primer end. The exonuclease and polymerase domains are located in separate regions of HsPOL $\gamma \mathrm{A}$ and the observed complementation between D274A and H1134A mutants shows that defects in different functional domains of POL $\gamma$ A can complement each other (Fig. 8c, compare lane 4 and 8 with lane 14). To accomplish this, complementation POL $\gamma \mathrm{A}$ must frequently go on and off the template during exonucleolytic proofreading and elongation.

\section{Discussion}

Faithful replication has long been assumed essential for mtDNA to prevent the accumulation of deleterious mutations in the absence of recombination. In addition, a yet not fully resolved mechanism during embryogenesis ensures that only a limited number of mtDNA molecules are transmitted through the female germline to the next generation. Flies seem to be more susceptible to impaired mtDNA maintenance than the mutator mice, because the majority of flies homozygous for the exo ${ }^{-}$DmPOL $\gamma \mathrm{A}$ did not pass the L3 larval stage, despite carrying approximately threefold less mtDNA mutations than mtDNA mutator mice ${ }^{41-43,53}$. 
a
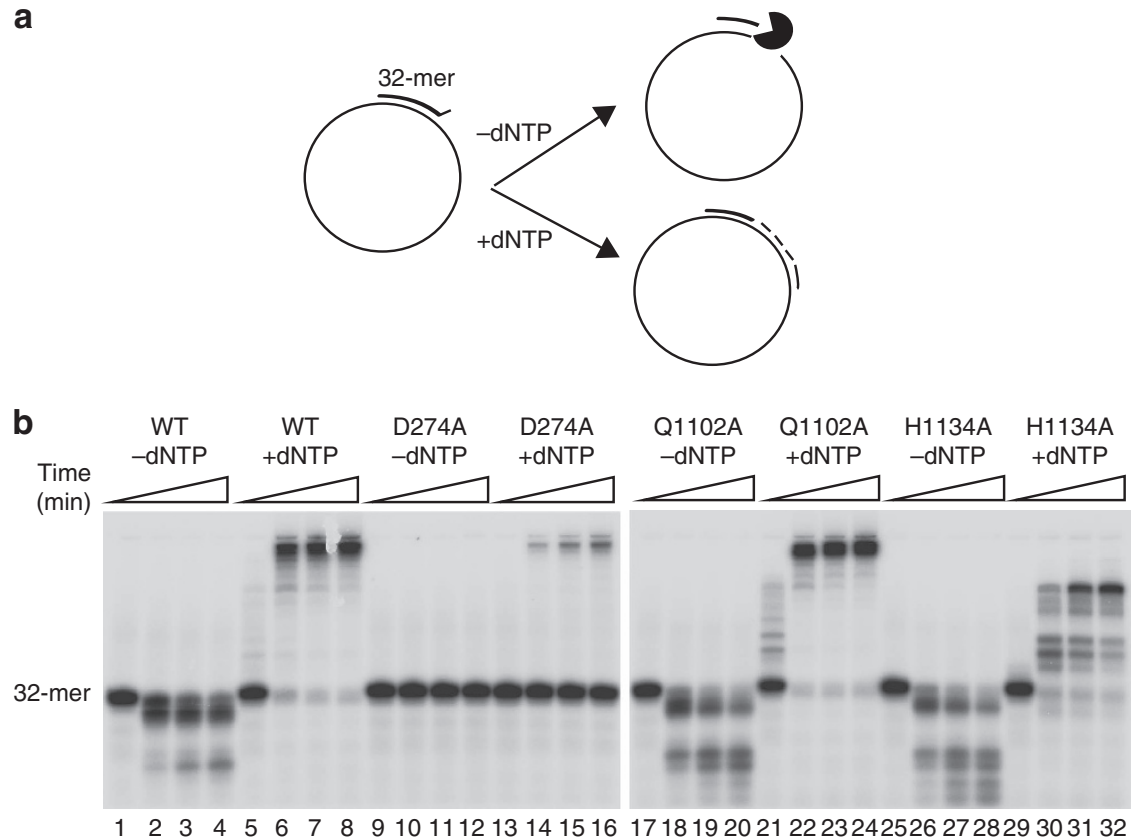

C

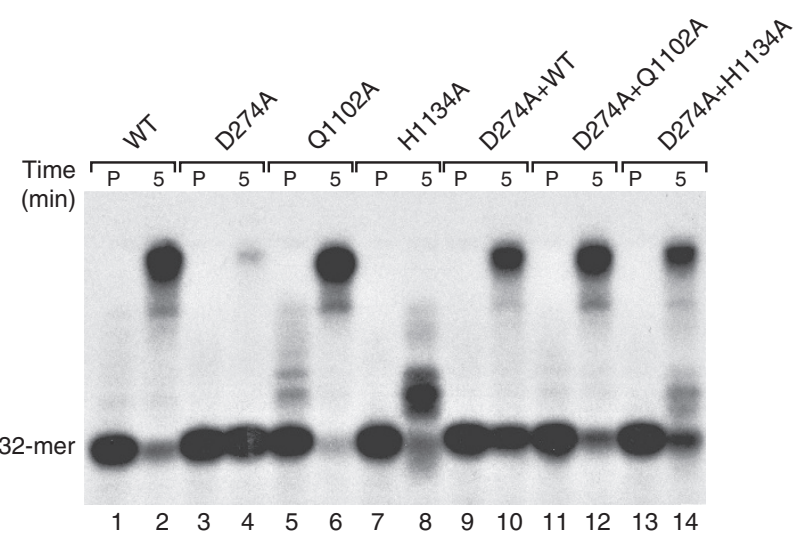

Figure 8 | Mutant recombinant HsPOL $\gamma$ A proteins can complement each other in in vitro assays. (a) Schematic overview of the HsPOL $\gamma \mathrm{A}$ in vitro proofreading and polymerase activity assays. The template used in these assays consisted of a $5^{\prime}{ }^{32} \mathrm{P}$-labelled 32 -mer annealed to single-stranded pBluescript SK $(+)$ creating one-nucleotide mismatch at the $3^{\prime}$-end. In the absence of dNTPs, HsPOL $\gamma$ A will backtrack ( $3^{\prime}$ to $5^{\prime}$ direction), degrading the oligonucleotide whereas in the presence of dNTPs the polymerase will proofread the mismatch and polymerize from the $3^{\prime}$-end. (b) The proofreadingdeficient D274A had no exonuclease activity (lane 9-12), which led to impaired ability to continue DNA synthesis from the mismatched primer end (lane 13-16). The Q1102A mutant showed both extensive proofreading (lane 17-20) and polymerase activities (lane 21-24) comparable to the WT enzyme (lane 1-8). The H1134A mutant had extensive exonuclease activity (lane 25-28) but was a poor polymerase only able to copy a short stretch of DNA (lane 29-32). Triangles indicate elapsed time with 15 min being the end point. (c) The D274A and H1134A mutants were not able to copy DNA alone from the mismatched primer end (lanes 4 and 8, respectively). However, by mixing the two mutant proteins we observed a synergistic effect (lane 14) suggesting cooperation at the mismatched primer-end. The cooperation of D274A with WT and Q1102A is not as pronounced as in this assay (lane 10 and 12). ' $P$ ' means the samples were pre-incubated on ice for $5 \mathrm{~min}$ and then stopped. Reactions labelled ' 5 ' were also pre-incubated on ice for 5 min but followed by a 5 -min incubation at $37^{\circ} \mathrm{C}$ before stopping the reaction. All experiments were repeated at least three time.

Similar to the situation in mtDNA mutator mice, the majority of mtDNA mutations accumulated during early developmental stages also in flies. The finding of arrested development, caused by high levels of mtDNA mutations or mtDNA depletion, show that mitochondrial function is important to allow larvae to proceed to the pupal stage. Interestingly, heterozygous exo ${ }^{-}$flies have lower mtDNA mutation levels than homozygous exo ${ }^{-}$larva and no obvious phenotype. These findings argue that mtDNA mutations will only impair development if the mtDNA mutation levels exceed a critical threshold. The mutational pattern observed in the exo ${ }^{-}$flies is analogous to the pattern in mtDNA mutator mice and consists mainly of transition mutations. The majority of mtDNA mutations are transitions also in wild animals and humans, which is consistent with the hypothesis that most of the mtDNA variation is created by replicative errors rather than by unrepaired base lesions caused by oxidation or other types of damage 22,24 .

In accordance with results from mtDNA mutator mice 22,42 , homozygous exo ${ }^{-}$larvae contained linear mtDNA molecules with deletions. In mice, the linear deleted mtDNA molecule spans the major arc between both origins of replication, and the levels of deleted mtDNA does not increase with time, which suggests they are formed by abortive replication of the full-length mtDNA molecule and are not themselves templates for mtDNA replication. We have recently demonstrated that the exonuclease activity of mammalian POL $\gamma$ is required to create ligatable ends at 
the termination of replication ${ }^{54}$. Inactivation of the exonuclease activity causes the appearance of unligated nicks at the origins of replication, which leads to linear deletions during subsequent rounds of mtDNA synthesis. The linear deletion observed in mouse therefore defines the location of the strand-specific origins for mtDNA synthesis ${ }^{54}$. In flies, the origins of mtDNA replication have not yet been defined, although the noncoding A + T-rich control region has been proposed to contain both origins in arthropods $^{52}$. In the homozygous exo ${ }^{-}$larvae, we mapped the ends of the linear deletions to the $\mathrm{A}+\mathrm{T}$-rich region. Our observation of linear deletions and the location of their ends therefore strongly suggests that also fly mtDNA, similar to mammalian mtDNA, has a dedicated origin of replication for each strand of mtDNA, and that these origins are located in the non-coding A + T-rich region of fly mtDNA.

Further, our results suggest that flies have a wider bottleneck during transmission of mtDNA through the maternal germline than mammals ${ }^{29}$. In mice, continuous breeding maternal lineages of heterozygous mtDNA mutator mice results in rapid accumulation of mtDNA mutations within a few generations ${ }^{29}$, whereas similar crosses of heterozygous exo ${ }^{-}$flies required six generations before an additional increase of the mtDNA mutation load could be observed. Interestingly, we observed that compound heterozygous flies showed a much more rapid clonal expansion of mtDNA mutations than exo ${ }^{-}$heterozygous flies. This is likely due to a post-fertilization bottleneck as the polymerase-deficient high-fidelity allele may reduce mtDNA copy number. Similar observations were made in the mouse, where mtDNA copy number during early development is reduced in heterozygous TFAM KO mice ${ }^{49}$. It is thus tempting to suggest that while the mammalian mitochondrial bottleneck results in low variation, flies transmit a larger number of mtDNA copies ${ }^{55}$ and thus tolerate more somatic mtDNA variation. The short lifespan of flies may thus not have led to the selection of a tight bottleneck during evolution.

Surprisingly, our results from the complementation studies suggest that the mtDNA polymerase can go on and off the template during replication and that, despite being incapable of replicating mtDNA in an efficient manner, the pol ${ }^{-}$variants may have been able to repair some of the replication errors introduced by the exo ${ }^{-}$mutant. This observation could very well explain the molecular nature behind disease-causing dominant versus recessive mutations in the $P O L \gamma A$ gene in humans. Several autosomal recessive mutations have been described and one of the most common is the A467T mutation, which has been found alone or in trans with other mutations in the same gene ${ }^{56}$. In vitro studies suggest that only $4 \%$ of the polymerase activity remains in A467T mutant alleles ${ }^{57}$. Heterozygous carriers are healthy, showing that the WT allele can complement the defect. In contrast, autosomal dominant mutations in the POL $\gamma A$ such as the Y955C leading to progressive external ophthalmoplegia (adPEO) stalls mtDNA replication at specific sites in vitro ${ }^{58}$. Autosomal dominant mutations thus might compete with the WT allele preventing full replication, and thereby cause mtDNA deletions or depletion.

In conclusion, we show here that mtDNA mutations transmitted through the fly germline are clonally expanded and they will severely impact fly development when they are present above a certain threshold. Furthermore, we provide genetic evidence that two Pol $\gamma A$ alleles, mutated in different domains, can functionally complement each other in vivo and in vitro, suggesting that mtDNA polymerase can re-initiate mtDNA replication after an aborted replicative cycle. Finally, our data suggest that the exonuclease activity of POL $\gamma \mathrm{A}$ is required for mtDNA replication to proceed past the origins of replication.

\section{Methods}

Expression and purification of recombinant human proteins. Mutagenesis of the human POL $\gamma$ A variants was carried out using the QuickChange Lightning Site-directed mutagenesis kit according to the provided protocol (Agilent). The presence of HsPOL $\gamma$ A mutations was confirmed by sequencing (Eurofins MWG Operon). Protein expression and purification of the different POL $\gamma \mathrm{A}$ variants, POL $\gamma B$, TWINKLE and mtSSB were done as previously described ${ }^{8}$. The concentrations of the HsPOL $\gamma \mathrm{A}$ mutants were determined by quantification from SDS-PAGE using WT HsPOL $\gamma$ A as reference.

EMSA and coupled exonuclease/polymerase assays. DNA affinity of POL $\gamma$ to a primer template was assayed using EMSA ${ }^{59}$. The reactions contained $10 \mathrm{fmol}$ DNA template and the indicated holoenzyme (POL $\gamma \mathrm{A} / \mathrm{POL} \gamma \mathrm{B}$ complex) concentrations in the presence of $300 \mu \mathrm{M}$ ddGTP and $3 \mathrm{mM}$ dCTP. The reactions were run on $6 \%$ native polyacrylamide gels in $0.5 \times \mathrm{TBE}$ for $35 \mathrm{~min}$ at $180 \mathrm{~V}$ and visualized by autoradiography. Each experiment was repeated three times. Band intensities representing unbound and bound DNA were quantified using Fujifilm Multi Gauge V3.1 software. The fraction of bound DNA was determined from the background-subtracted signal intensities using the expression: bound/(bound + unbound). The fraction of DNA bound in each reaction was plotted versus the concentration of POL $\gamma$. Data were fit with the binding equation $($ Fraction bound $=(\operatorname{MaxB} \times[\mathrm{POL} \gamma]) /(\mathrm{MaxB}+[\mathrm{POL} \gamma])$ using EXCELs Add-in 'Solver' to perform non-linear regression and obtain values for $\mathrm{Kd}$ (as the value corresponding to the midpoint of MaxB) and using MaxB set to 1 (the fraction bound at which the data plateaus). The EMSA substrate was also utilized to examine the polymerization and the $3^{\prime}$ to $5^{\prime}$ exonuclease activities of POL $\gamma \mathrm{A}$ as previously described ${ }^{60}$ but with $150 \mathrm{fmol}$ of the indicated POL $\gamma \mathrm{A}$ variant and $600 \mathrm{fmol}$ of POL $\gamma \mathrm{B}$. The reactions were analysed on a $15 \%$ denaturing polyacrylamide gel electrophoresis in $1 \times \mathrm{TBE}$ and visualized by autoradiography.

Second strand DNA synthesis. A ${ }^{32}$ P-labelled 32-mer (5'-CTATCTCAGCGAT CTGTCTATTTCGTTCATCC- $3^{\prime}$ ) was annealed to SS pBluescript SK $(+)$. DNA synthesis reactions were performed as described previously and were incubated at $37^{\circ} \mathrm{C}$ for the times indicated ${ }^{60}$. The products were analysed by electrophoresis in a $0.9 \%$ agarose gel and visualized by autoradiography.

In vitro rolling circle DNA replication. An oligonucleotide consisting of 70 nucleotides $\left(5^{\prime}-42[\mathrm{~T}]\right.$-ATCTCAGCGATCTGTCTATTTCGTTCAT-3') was hybridized to a SS pBluescript SK $(+)$ followed by one cycle of polymerization with KOD polymerase (Novagen) to produce an $\sim 3$-kb double-stranded template with a preformed replication fork. The reactions were performed as described ${ }^{61}$, and were incubated at $37^{\circ} \mathrm{C}$ for times indicated $(0,5,15,30$ and $60 \mathrm{~min})$. The products were analysed by electrophoresis in a $0.8 \%$ denaturing agarose gel and visualized by autoradiography.

$\mathbf{3}^{\prime}-\mathbf{5}^{\prime}$ exonuclease activity. A $5^{\prime}{ }^{32} \mathrm{P}$-labelled 32-mer (5'-CTATCTCAGCGAT CTGTCTATTTCGTTCATCG-3') with a one-nucleotide mismatch at the $3^{\prime}$-end was annealed to SS pBluescript $\mathrm{SK}(+)$. The reactions were performed as in the second strand DNA synthesis assay but in the absence or presence of dNTPs and with indicated POL $\gamma \mathrm{A}$ variant. The reactions were incubated at $37^{\circ} \mathrm{C}$ for the times indicated. The samples were analysed by electrophoresis in a $20 \%$ denaturing PAGE and visualized by autoradiography.

Processivity assays. Assays were performed by mixing $10 \mu \mathrm{l}$ of reaction mixture A with $10 \mu \mathrm{l}$ of reaction mixture B followed by incubation at $37^{\circ} \mathrm{C}$ for $10 \mathrm{~min}$. Reaction mixture A contained $25 \mathrm{fmol}$ template as described in Fig. 1d for second strand synthesis, $50 \mathrm{fmol}$ of the indicated POL $\gamma \mathrm{A}$ versions and $200 \mathrm{fmol}$ of POL $\gamma \mathrm{B}$, $1 \mathrm{mM}$ DTT, $25 \mathrm{mM}$ TrisHCl pH 7.8 and $0.1 \mathrm{mg} \mathrm{ml}^{-1}$ BSA. Reaction mixture B contained $10 \mathrm{mM} \mathrm{MgCl}_{2}, 100 \mu \mathrm{M} \mathrm{dNTP}$ and $0,001 \mathrm{mg} \mathrm{ml}^{-1}$ heparin (heparin was omitted in reactions that allowed for multiple binding events to occur). Reactions were stopped by the addition of $20 \mu \mathrm{l}$ of $95 \%$ formamide, $20 \mathrm{mM}$ EDTA and $0.1 \%$ bromophenol blue. The samples were analysed by electrophoresis on $12 \%$ denaturing PAGE.

Drosophila stocks and maintenance. Deficiency lines (Df(2L)Exel7059, Df(2L)BSC252, Df(2L)BSC694) and hypomorphic tamas alleles tam3 and tam4 were obtained from Bloomington stock center. TFAM knockdown lines (\#1: ID107191 and \#2: ID37819) were purchased from the Vienna Drosophila RNAi center (VDRC, Austria). All genomically engineered fly strains were constantly backcrossed into a white Dahomey Wolbachia-free (wDahT) WT strain to avoid accumulation of mtDNA mutations unless stated otherwise. All fly stocks were maintained at $25^{\circ} \mathrm{C}$ on a $12: 12 \mathrm{~h}$ light/dark cycle with $65 \%$ humidity and fed on a sugar/yeast/agar (SYA) $\operatorname{diet}^{62}$.

Generation of genomically engineered DmPOL $\gamma \mathbf{A}$ flies. Experimental flies were generated by genomic engineering in a two-step process ${ }^{48}$. In the first step, we generated a DmPOL $\gamma \mathrm{A}$ KO founder line by replacing the endogenous DmPOL $\gamma \mathrm{A}$ 
gene (tamas) with an attP site by ends-out homologous recombination. The attP site was then used in the second step to reintroduce wild-type and mutated versions of the tamas gene by $\Phi 31$-mediated recombination.

For the ends-out homologous recombination donor construct $\sim 4 \mathrm{~kb}$ of $5^{\prime}$ and $3^{\prime}$ flanking sequences of the tamas gene were cloned into the pBlueScript II $\mathrm{SK}(+)$ vector (Stratagene) by ET recombination using gene-specific primers (Supplementary Table 6) and a tamas BAC clone (RP98-30I21, BACPAC Resource Center, Oakland, California). $5^{\prime}$ and $3^{\prime}$ homologous arms were sequence verified and subsequently cloned into the pGXattP targeting vector ${ }^{48}$. Transgenic flies carrying the tamas pGXattP targeting vector were generated by P-elementmediated germ line transformation using the BestGene Drosophila embryo injection service. Crosses for ends-out homologous recombination were carried out according to the rapid targeting scheme ${ }^{63}$. Subsequently, the white $\mathrm{e}^{\mathrm{hs}}$ marker gene was genetically mapped and homologous recombination events were identified by PCR using primers PCR3 and PCR4 (Supplementary Table 6). Tamas KO flies were crossed to flies expressing a cre-recombinase to remove the white ${ }^{\text {hs }}$ marker gene ${ }^{48}$. Absence of the tamas gene was confirmed by PCR using primers PCR1 (Supplementary Table 6). The corresponding flies are referred to as tamas KO flies.

Rescue construct (Supplementary Fig. 3a) was amplified by PCR using primers PCR8 (Supplementary Table 6). Rescue construct was sequence verified and subsequently cloned using XhoI and AscI sites into the pGEattBGMR vector ${ }^{48}$. Mutagenesis of the WT tamas allele was carried out using the 'QuickChange Lightning Site-directed mutagenesis kit' according to the provided protocol and pGEattBGMR-Rescue construct was used as a template. Primers used for site-directed mutagenesis are indicated in Supplementary Table 6. All mutant tamas allelic variants were sequenced to ensure the absence of base substitutions. The presence of D263A, H1038A and/or Q1009A mutations was confirmed by sequencing. Mutant variants of the tamas gene were then injected into the embryos of tamas KO flies expressing $\Phi 31$ integrase by the in-house Drosophila transgenic core facility. Primers used to verify the precise re-introduction of tamas allelic variants into the native tamas locus were PCR5 and PCR6 (Supplementary Table 6). The absence of genomic rearrangements on re-introduction of tamas mutant alleles was confirmed by Southern blot analyses. Total DNA was isolated from the corresponding genotypes and digested with XhoI. A full-length complementary DNA (cDNA) of DmPOL $\gamma$ A was used as a probe and the signal was visualized by autoradiography.

To verify that the white ${ }^{\text {hs }}$ marker gene did not interfere with DmPOL $\gamma$ A function and induced lethality, all tamas mutant lines were crossed to flies expressing the cre-recombinase. Precise removal of the white ${ }^{\text {hs }}$ marker gene was confirmed by PCR using primers PCR5 and PCR7 (Supplementary Table 6). Tamas mutant flies with or without the white ${ }^{\text {hs }}$ marker gene showed similar survival, thus for all experiments tamas mutant flies carrying the white ${ }^{\text {hs }}$ marker gene were used.

Fly developmental time. To assess developmental time, flies were allowed to lay eggs on grape juice agar plates for $3 \mathrm{~h}$. For each genotype, 100 eggs were individually picked and placed into vials with SYA food. The number of eclosed flies was scored every $12 \mathrm{~h}$. At least five biological replicates were done for each genotype.

Developmental analysis of DmPOL $\gamma \mathbf{A}$ mutant flies. For assessing the percentage of DmPOL $\gamma$ A mutant flies entering L3 stage, flies were allowed to lay eggs on grape juice agar plates for $2 \mathrm{~h}$. For each genotype, 100 eggs were individually picked and placed into vials with SYA food. The number of L3 larvae of each genotype was scored 6 days AEL. At least six biological replicates were done for each genotype.

To assess the percentage of DmPOL $\gamma \mathrm{A}$ mutant flies entering the pupal stage, $50 \mathrm{~L} 3$ larvae were collected and transferred into vial with SYA food. The number of pupae was scored every $12 \mathrm{~h}$. At least three biological replicates were done for each genotype.

To assess the percentage of DmPOL $\gamma \mathrm{A}$ mutant flies entering the adult stage, $50 \mathrm{~L} 3$ larvae were collected and transferred into vial with SYA food. The number of adult flies was scored every $12 \mathrm{~h}$. At least three biological replicates were done for each genotype.

DNA isolation and qRT-PCR and Southern blot analyses. For relative mtDNA copy number determination, total DNA extractions were prepared from L3 larvae using DNeasy Blood and Tissue Kit (Qiagen). Five biological replicates, each with 10 larvae, were prepared for each genotype. Quantification of mtDNA levels was done using SYBR-Green qPCR analyses and primers targeting cytB and rpL32 (Supplementary Table 6). All data were normalized against wild type levels.

For Southern blot analysis, total DNA was extracted from 20-30 L3 larvae. Samples were homogenized with a tissue grinder in $400 \mu \mathrm{l}$ of buffer A (100 mM Tris- $\mathrm{HCl}, \mathrm{pH} 7.5 ; 100 \mathrm{Mm}$ EDTA; $100 \mathrm{mM} \mathrm{NaCl}$ and $0.5 \%$ SDS). After incubation at $65^{\circ} \mathrm{C}$ for $30 \mathrm{~min}, 800 \mu \mathrm{l}$ of Buffer B $(4 \mathrm{ml}$ of $5 \mathrm{M} \mathrm{KOAc}$ and $10 \mathrm{ml}$ of $6 \mathrm{M}$ lithium chloride) was added and samples were left on ice for $60 \mathrm{~min}$. After incubation, samples were centrifuged at $12,000 \mathrm{~g}$ for $15 \mathrm{~min}$ and supernatant was transferred into a new tube. About $540 \mu \mathrm{l}$ of isopropanol was added to the supernatant and samples were further centrifuged at $12000 \mathrm{~g}$ for $15 \mathrm{~min}$. Pellet was washed with $70 \%$ ethanol, dried and resuspended in $100 \mu \mathrm{l}$ nuclease-free water containing $20 \mu \mathrm{g} \mathrm{ml}^{-1}$ RNase A. After incubation at $37^{\circ} \mathrm{C}$ for $1 \mathrm{~h}$, samples were stored at $+4^{\circ} \mathrm{C}$.
Approximately, 1-3 $\mu \mathrm{g}$ of total DNA was cut using either EcoRV, PstI, NdeI, NsiI or StyI restriction endonuclease. Digestions were run on $0.8 \%$ agarose gels, and blotted to Hybond-N + membrane (Amersham Bioscience). ND2, COXI or $12 \mathrm{~S}$ rRNA were used as probes and signals were visualized by autoradiography. ${ }^{32} \mathrm{P}$-labelling of Southern probes was done according to manufacturer's instructions (Prime-IT II Random Prime Labeling Kit, Agilent). Primers used to prepare the mtDNA probes can be found in the Supplementary Table 6.

RNA isolation and quantitative RT-PCR. Total RNA was extracted from 5-day-old larvae using ToTALLY RNA isolation kit (Ambion). For expression analyses, RNA was DNase treated using Turbo DNA-free kit (Ambion) and reverse transcribed using High capacity cDNA Reverse Transcription kit (Applied Biosystems). The expression profiles were determined by quantitative reverse transcription-PCR (qRT-PCR) analyses with a 7900HT Fast Real Time PCR System (Applied Biosystems). Rpl32 was used as a normalization control $^{64}$. Taqman probes were provided by Applied Biosystems under following assay numbers: tamas (Dm01841857_g1); CG8978 (Dm01807408_g1); CG7833 (Dm01842615_g1); CG7811 (Dm01841741_g1); CG33650 (assay is not available anymore).

MtDNA mutation load analysis. Total DNA was extracted from whole larvae or from the thorax of adult male flies using the DNeasy Blood and Tissue Kit. The mtDNA mutation load was determined by PCR, cloning and sequencing as described elsewhere ${ }^{22,42,65}$. Fly mtDNA-specific primers were used to amplify a $1.2-\mathrm{kb}$ region encompassing Leu-transfer RNA and parts of COXI and COXII (nucleotide pair 2,194-3,382). An average number of 90 colonies were analysed per animal.

Biochemical evaluation of respiratory chain function. Five-day-old larvae were collected and the respiratory rates were measured as described by ref. 66. Briefly, $5 \mathrm{~L} 3$ larvae were dissected in $100 \mu \mathrm{l}$ of respiration buffer $(120 \mathrm{mM}$ sucrose, $50 \mathrm{mM}$ $\mathrm{KCl}, 20 \mathrm{mM}$ Tris- $\mathrm{HCl}, 4 \mathrm{mM} \mathrm{KH} \mathrm{KO}_{4}, 2 \mathrm{mM} \mathrm{MgCl}$, 1 mM EGTA, 0,01\% digitonin, $\mathrm{pH} 7.2$ ) and oxygen consumption was monitored at $27^{\circ} \mathrm{C}$ using oxygraph (OROBOROS). State 3 respiration was assessed by adding the following substrates: proline $(10 \mathrm{mM})$, pyruvate $(10 \mathrm{mM})$, malate $(5 \mathrm{mM})$, glutamate $(5 \mathrm{mM})$ and $\operatorname{ADP}(1 \mathrm{mM})$. State 4 respiration was assessed by adding oligomycin $\left(250 \mathrm{ng} \mathrm{ml}^{-1}\right)$ and uncoupled state was obtained by adding $1 \mu \mathrm{M}$ CCCP. Oxygen consumption rates were normalized to total protein content quantified by the Bradford method (Sigma).

Statistical analysis. Statistical analyses were performed using GraphPad Prism 5.03 (GraphPad Prism Software, Inc). For statistical analyses of qPCR analyses, qRT-PCR analyses and mtDNA mutation loads one-way ANOVA with Dunnett's test post hoc was used unless stated otherwise. For statistical analyses of OXPHOS function Mann-Whitney two-tailed test was used. For statistical analyses of body weight, Tukey's multiple comparison test was used. All data are presented as mean \pm s.d.

\section{References}

1. Kukat, C. \& Larsson, N.-G. mtDNA makes a U-turn for the mitochondrial nucleoid. Trends Cell Biol. 23, 457-463 (2013).

2. Kukat, C. et al. Cross-strand binding of TFAM to a single mtDNA molecule forms the mitochondrial nucleoid. Proc. Natl Acad. Sci. USA 112, 11288-11293 (2015).

3. Farge, G. et al. In vitro-reconstituted nucleoids can block mitochondrial DNA replication and transcription. Cell Rep. 8, 66-74 (2014).

4. Ropp, P. A. \& Copeland, W. C. Cloning and characterization of the human mitochondrial DNA polymerase, DNA polymerase gamma. Genomics 36, 449-458 (1996).

5. Kaguni, L. S. DNA polymerase $\gamma$, the mitochondrial replicase 1. Annu. Rev. Biochem. 73, 293-320 (2004).

6. Wang, Y. X., Farr, C. L. \& Kaguni, L. S. Accessory subunit of mitochondrial DNA polymerase from Drosophila embryos-Cloning, molecular analysis, and association in the native enzyme. J. Biol. Chem. 272, 13640-13646 (1997).

7. Carrodeguas, J. A., Theis, K., Bogenhagen, D. F. \& Kisker, C. Crystal structure and deletion analysis show that the accessory subunit of mammalian DNA polymerase gamma, Pol gamma B, functions as a homodimer. Mol. Cell 7, 43-54 (2001)

8. Korhonen, J. A., Pham, X. H., Pellegrini, M. \& Falkenberg, M. Reconstitution of a minimal mtDNA replisome in vitro. EMBO J. 23, 2423-2429 (2004)

9. Hance, N., Ekstrand, M. I. \& Trifunovic, A. Mitochondrial DNA polymerase gamma is essential for mammalian embryogenesis. Hum. Mol. Genet. 14, 1775-1783 (2005).

10. Humble, M. M. et al. Polg2 is essential for mammalian embryogenesis and is required for mtDNA maintenance. Hum. Mol. Genet. 22, 1017-1025 (2013). 
11. Milenkovic, D. et al. TWINKLE is an essential mitochondrial helicase required for synthesis of nascent D-loop strands and complete mtDNA replication. Hum. Mol. Genet. 22, 1983-1993 (2013).

12. Lightowlers, R. N. \& Chrzanowska-Lightowlers, Z. M. A. Exploring our origins - the importance of OriL in mtDNA maintenance and replication. EMBO Rep. 13, 1038-1039 (2012).

13. Clayton, D. A. Replication of animal mitochondrial DNA. Cell 28, 693-705 (1982).

14. Wanrooij, S. et al. In vivo mutagenesis reveals that OriL is essential for mitochondrial DNA replication. EMBO Rep. 13, 1130-1137 (2012).

15. Wanrooij, S. et al. Human mitochondrial RNA polymerase primes lagging-strand DNA synthesis in vitro. Proc. Natl Acad. Sci. USA 105, 11122-11127 (2008).

16. Kühl, I. et al. POLRMT does not transcribe nuclear genes. Nature 514, E7-11 (2014).

17. Mita, S. et al. Recombination via flanking direct repeats is a major cause of large-scale deletions of human mitochondrial-DNA. Nucleic Acids Res. 18, 561-567 (1990).

18. Moraes, C. T. et al. Replication-competent human mitochondrial-DNA lacking the heavy-strand promoter region. Mol. Cell. Biol. 11, 1631-1637 (1991).

19. Fusté, J. M. et al. In vivo occupancy of mitochondrial single-stranded DNA binding protein supports the strand displacement mode of DNA replication. PLoS Genet. 10, e1004832 (2014).

20. Wallace, D. C. Mitochondrial diseases in man and mouse. Science 283, 1482-1488 (1999).

21. Stewart, J. B. et al. Strong purifying selection in transmission of mammalian mitochondrial DNA. PLoS Biol. 6, e10 (2008).

22. Ameur, A. et al. Ultra-deep sequencing of mouse mitochondrial DNA: Mutational patterns and their origins. PLoS Genet. 7, e1002028 (2011).

23. Kennedy, S. R., Salk, J. J., Schmitt, M. W. \& Loeb, L. A. Ultra-sensitive sequencing reveals an age-related increase in somatic mitochondrial mutations that are inconsistent with oxidative damage. PLoS Genet. 9, e1003794 (2013).

24. Itsara, L. S. et al. Oxidative stress is not a major contributor to somatic mitochondrial DNA mutations. PLoS Genet. 10, e1003974 (2014).

25. Copeland, W. C. Defects of mitochondrial DNA replication. J. Child Neurol. 29, 1216-1224 (2014).

26. Bogenhagen, D. \& Clayton, D. A. Mouse L cell mitochondrial DNA molecules are selected randomly for replication throughout the cell cycle. Cell 11, 719-727 (1977).

27. Larsson, N.-G. Somatic mitochondrial DNA mutations in mammalian aging. Annu. Rev. Biochem. 79, 683-706 (2010).

28. Hagström, E., Freyer, C., Battersby, B. J., Stewart, J. B. \& Larsson, N.-G. No recombination of mtDNA after heteroplasmy for 50 generations in the mouse maternal germline. Nucleic Acids Res. 42, 1111-1116 (2014).

29. Stewart, J. B. \& Larsson, N.-G. Keeping mtDNA in shape between generations. PLoS Genet. 10, e1004670 (2014).

30. Freyer, C. et al. Variation in germline mtDNA heteroplasmy is determined prenatally but modified during subsequent transmission. Nat. Genet. 44, 1282-1285 (2012).

31. Hill, J. H., Chen, Z. \& Xu, H. Selective propagation of functional mitochondrial DNA during oogenesis restricts the transmission of a deleterious mitochondrial variant. Nat. Genet. 46, 389-392 (2014).

32. Fan, W. et al. A mouse model of mitochondrial disease reveals germline selection against severe mtDNA mutations. Science 319, 958-962 (2008).

33. Elson, J. L., Turnbull, D. M. \& Howell, N. Comparative genomics and the evolution of human mitochondrial DNA: assessing the effects of selection. Am. J. Hum. Genet. 74, 229-238 (2004).

34. Soares, P., Abrantes, D., Rito, T., Thomson, N. \& Radivojac, P. Evaluating purifying selection in the mitochondrial DNA of various mammalian species. PLoS ONE 8, e58993 (2013).

35. Hauswirth, W. W. \& Laipis, P. J. Mitochondrial DNA polymorphism in a maternal lineage of Holstein cows. Proc. Natl Acad. Sci. USA 79, 4686-4690 (1982).

36. Jenuth, J. P., Peterson, A. C., Fu, K. \& Shoubridge, E. A. Random genetic drift in the female germline explains the rapid segregation of mammalian mitochondrial DNA. Nat. Genet. 14, 146-151 (1996).

37. Cree, L. M. et al. A reduction of mitochondrial DNA molecules during embryogenesis explains the rapid segregation of genotypes. Nature 40, 249-254 (2008).

38. Wai, T., Teoli, D. \& Shoubridge, E. A. The mitochondrial DNA genetic bottleneck results from replication of a subpopulation of genomes. Nat. Genet. 40, 1484-1488 (2008).

39. Ma, H., Xu, H. \& O'Farrell, P. H. Transmission of mitochondrial mutations and action of purifying selection in Drosophila melanogaster. Nat. Genet. 46, 393-397 (2014).

40. Stewart, J. B., Freyer, C., Elson, J. L. \& Larsson, N.-G. Purifying selection of mtDNA and its implications for understanding evolution and mitochondrial disease. Nat. Rev. Genet. 9, 657-662 (2008).

41. Ross, J. M. et al. Germline mitochondrial DNA mutations aggravate ageing and can impair brain development. Nature 501, 412-415 (2013).
42. Trifunovic, A. et al. Premature ageing in mice expressing defective mitochondrial DNA polymerase. Nature 429, 417-423 (2004).

43. Kujoth, G. C. Mitochondrial DNA mutations, oxidative stress, and apoptosis in mammalian aging. Science 309, 481-484 (2005).

44. Minnick, D. T. D. et al. Side chains that influence fidelity at the polymerase active site of Escherichia coli DNA polymerase I (Klenow fragment). J. Biol. Chem. 274, 3067-3075 (1999).

45. Foury, F. \& Szczepanowska, K. Antimutator alleles of yeast DNA polymerase gamma modulate the balance between DNA synthesis and excision. PLoS ONE 6, e27847 (2011).

46. Vanderstraeten, S., Van den Brule, S., Hu, J. P. \& Foury, F. The role of 3 ' -5 ' exonucleolytic proofreading and mismatch repair in yeast mitochondrial DNA error avoidance. J. Biol. Chem. 273, 23690-23697 (1998).

47. Lefai, E. et al. Overexpression of the catalytic subunit of DNA polymerase gamma results in depletion of mitochondrial DNA in Drosophila melanogaster. Mol. Gen. Genet. 264, 37-46 (2000).

48. Huang, J., Zhou, W., Dong, W., Watson, A. M. \& Hong, Y. From the Cover: Directed, efficient, and versatile modifications of the Drosophila genome by genomic engineering. Proc. Natl Acad. Sci. USA 106, $8284-8289$ (2009)

49. Larsson, N. G. et al. Mitochondrial transcription factor A is necessary for mtDNA maintenance and embryogenesis in mice. Nat. Genet. 18, 231-236 (1998).

50. Bratic, I. et al. Mitochondrial DNA level, but not active replicase, is essential for Caenorhabditis elegans development. Nucleic Acids Res. 37, 1817-1828 (2009)

51. Iyengar, B., Roote, J. \& Campos, A. R. The tamas gene, identified as a mutation that disrupts larval behavior in Drosophila melanogaster, codes for the mitochondrial DNA polymerase catalytic subunit (DNApol- ${ }^{-}$amma125). Genetics 153, 1809-1824 (1999).

52. Saito, S., Tamura, K. \& Aotsuka, T. Replication origin of mitochondrial DNA in insects. Genetics 171, 1695-1705 (2005).

53. Trifunovic, A. et al. Somatic mtDNA mutations cause aging phenotypes without affecting reactive oxygen species production. Proc. Natl Acad. Sci. USA 102, 17993-17998 (2005).

54. Macao, B. et al. The exonuclease activity of DNA polymerase $\gamma$ is required for ligation during mitochondrial DNA replication. Nat. Commun. 6, 7303 (2015).

55. Wolff, J. N. \& Gemmell, N. J. Mitochondria, maternal inheritance, and asymmetric fitness: why males die younger. Bioessays 35, 93-99 (2013).

56. Tzoulis, C. et al. The spectrum of clinical disease caused by the A467T and W748SPOLG mutations: a study of 26 cases. Brain 129, 1685-1692 (2006).

57. Chan, S. S. L., Longley, M. J. \& Copeland, W. C. The common A467T mutation in the human mitochondrial DNA polymerase (POLG) compromises catalytic efficiency and interaction with the accessory subunit. J. Biol. Chem. 280, 31341-31346 (2005).

58. Atanassova, N. et al. Sequence-specific stalling of DNA polymerase $\gamma$ and the effects of mutations causing progressive ophthalmoplegia. Hum. Mol. Genet. 20, 1212-1223 (2011).

59. Farge, G., Pham, X. H., Holmlund, T., Khorostov, I. \& Falkenberg, M. The accessory subunit B of DNA polymerase is required for mitochondrial replisome function. Nucleic Acids Res. 35, 902-911 (2007).

60. Roos, S. et al. Subnormal levels of POLA cause inefficient initiation of light-strand DNA synthesis and lead to mitochondrial DNA deletions and autosomal dominant progressive external ophthalmoplegia. Hum. Mol. Genet. 22, 2411-2422 (2013).

61. Fusté, J. M. et al. Mitochondrial RNA polymerase is needed for activation of the origin of light-strand DNA replication. Mol. Cell 37, 67-78 (2010).

62. Bass, T. M. et al. Optimization of dietary restriction protocols in Drosophila. J. Gerontol. A Biol. Sci. Med. Sci. 62, 1071-1081 (2007).

63. Rong, Y. S. \& Golic, K. G. A targeted gene knockout in Drosophila. Genetics 157, 1307-1312 (2001).

64. Wredenberg, A. et al. MTERF3 regulates mitochondrial ribosome biogenesis in invertebrates and mammals. PLoS Genet. 9, e1003178 (2013).

65. Wanrooij, P. H. et al. A hybrid G-quadruplex structure formed between RNA and DNA explains the extraordinary stability of the mitochondrial R-loop. Nucleic Acids Res. 40, 10334-10344 (2012).

66. Wredenberg, A. et al. Increased mitochondrial mass in mitochondrial myopathy mice. Proc. Natl Acad. Sci. USA 99, 15066-15071 (2002).

\section{Acknowledgements}

This study was supported by a grant from the Swedish Research Council (2013-2859) to N.-G.L. A.W. is supported by the Swedish foundation for strategic research (ICA12-0017), the Swedish research council (2012-2571) and is a Ragnar Söderberg fellow (K0176-2012). T.E.S.K. was supported by Finnish Cultural Foundation (00140383). We thank to Bloomington Stock Center for providing flies.

\section{Author contributions}

A.B., T.E.S.K., S.G., L.P, M.F., A.W. and N-G.L. conceived and designed the study. A.B., T.E.S.K., B.M., S.G., T.S., J.B.S, F.B. and A.W. performed the experiments. 
A.B., T.E.S.K., B.M., S.G., T.S., J.B.S., M.F. and A.W. analysed the data. J.D. performed embryo injections. A.B., T.E.S.K., B.M., M.F., A.W. and N.-G.L. wrote the manuscript.

\section{Additional information}

Supplementary Information accompanies this paper at http://www.nature.com/ naturecommunications

Competing financial interests: The authors declare no competing financial interests.

Reprints and permission information is available online at http://npg.nature.com/ reprintsandpermissions/
How to cite this article: Bratic, A. et al. Complementation between polymeraseand exonuclease-deficient mitochondrial DNA polymerase mutants in genomically engineered flies. Nat. Commun. 6:8808 doi: 10.1038/ncomms9808 (2015).

(c) (i) This work is licensed under a Creative Commons Attribution 4.0 International License. The images or other third party material in this article are included in the article's Creative Commons license, unless indicated otherwise in the credit line; if the material is not included under the Creative Commons license, users will need to obtain permission from the license holder to reproduce the material. To view a copy of this license, visit http://creativecommons.org/licenses/by/4.0/ 